\title{
Small molecules activators of the Nrf2-HO-1 antioxidant axis modulate heme metabolism and inflammation in BV2 microglia cells
}

\author{
Roberta Foresti $^{\text {a,b, }}$, Sandip K. Bains ${ }^{\mathrm{c}}$, Tamil Selvi Pitchumony ${ }^{\mathrm{c}}$, Lisandra E. de Castro \\ Brás $^{c, \$}$, Filippo Drago ${ }^{d}$, Jean-Luc Dubois-Randéa,e, \\ Claudio Bucolo ${ }^{\mathrm{d}}$ and Roberto Motterlini ${ }^{\mathrm{ab}, \mathrm{e}}$ \\ âniversité Paris-Est, UMR_S955, UPEC, F-94000, Créteil, France \\ ${ }^{\mathrm{b}}$ Inserm U955, Equipe 3, F-94000, Créteil, France \\ ${ }^{c}$ Department of Drug Discovery and Development, Italian Institute of Technology, 16163 \\ Genova, Italy \\ ${ }^{\mathrm{d}}$ Department of Clinical and Molecular Biomedicine, Section of Pharmacology and \\ Biochemistry, University of Catania, 95125 Catania, Italy \\ eAP-HP, Hôpital Henri Mondor, Service de Cardiologie 1, F-94010 Créteil, France
}

\begin{abstract}
* To whom correspondence may be addressed: Roberta Foresti or Roberto Motterlini, Inserm U955, Equipe 3, F-94000, Créteil, France, Tel : +33 $14981 \quad 36$ 37, Email: roberta.foresti@inserm.fr or roberto.motterlini@inserm.fr; ${ }^{\S}$ Current address: Jackson Center for Heart Research at UMMC, Jackson, Mississippi, USA
\end{abstract}

List of non-standard abbreviations: ALAS 1, delta-aminolevulinate synthase 1; ARE, antioxidant responsive element; BVR, biliverdin reductase; $\mathrm{tBH}$, tert-Butyl hydroquinone; $\mathrm{CA}$, carnosol; CAA, carnosic acid; CoPP, Cobalt Protoporphyrin IX; CO-RMs, carbon monoxidereleasing molecules; DHC, 2,2-Dihydroxychalcone; DMF, dimethyl fumarate; H, hemin; HO-1, heme oxygenase-1; Keap-1, Kelch-like ECH-associated protein-1; INF- $\gamma$, interferon- $\gamma$; ISL, isoliquiritigenin; LPS, lipopolysaccharide; Nrf2, HF-E2 p45-related factor 2; PGE2, prostglandin E2; S, sulforaphane; SC, supercurcumin; shRNA, small hairpin RNA; SnPPIX, tin protoporphyrin IX; TNF- $\alpha$, tumor necrosis factor alpha. 


\begin{abstract}
The nuclear factor erythroid derived 2-related factor 2 (Nrf2) and the antioxidant protein heme oxygenase-1 (HO-1) are crucial components of the cellular stress response. These two systems work together to combat oxidative stress and inflammation and are attractive drug targets for counteracting different pathologies, including neuroinflammation. We aimed to identify the most effective Nrf2/HO-1 activators that modulate the inflammatory response in microglia cells. In the present study, we searched the literature and selected 56 compounds reported to activate Nrf 2 or HO-1 and analysed them for HO-1 induction at 6 and $24 \mathrm{~h}$ and cytotoxicity in BV2 microglial cells in vitro. Approximately 20 compounds up-regulated HO-1 at the concentrations tested (5 to $20 \mu \mathrm{M}$ ) with carnosol, supercurcumin, cobalt protoporphyrin-IX and dimethyl fumarate exhibiting the best induction/low cytotoxicity profile. Up-regulation of HO-1 by some compounds resulted in increased cellular bilirubin levels but did not augment the expression of proteins involved in heme synthesis (ALAS 1) or biliverdin reductase. Bilirubin production by HO-1 inducers correlated with their potency in inhibiting nitrite production after challenge with interferon- $\gamma$ (INF- $\gamma)$ or lipopolysaccharide (LPS). The compounds down-regulated the inflammatory response (TNF- $\alpha$, PGE2 and nitrite) more strongly in cells challenged with INF- $\gamma$ than LPS, and silencing HO-1 or Nrf2 with shRNA differentially affected the levels of inflammatory markers. These findings indicate that some small activators of $\mathrm{Nrf} / \mathrm{HO}-1$ are effective modulators of microglia inflammation and highlight the chemical scaffolds that can serve for the synthesis of potent new derivatives to counteract neuroinflammation and neurodegeneration.
\end{abstract}


Keywords: BV2 microglia; Nrf2; HO-1; small molecule activators; heme metabolism; inflammation.

\section{Introduction}

Inflammation in the brain is considered to be a significant cause underlying the development of neurodegenerative disorders [1]. Microglia, the resident macrophages of the brain, reacts to systemic and local inflammation as well as other stressful stimuli by activating a stress response. This response involves additional production of inflammatory molecules, reactive oxygen species and other mediators that may lead to detrimental effects. However, microglia activation is also important for stimulation of repair mechanisms and protection of neurons, indicating the dual role covered by microglial cells and emphasizing the delicate balance existing between pro- and antiinflammatory responses in the context of brain pathophysiology.

In common with other cell types, microglia expresses the Nrf2 and heme oxygenase-1 (HO1) systems, which are essential for counteracting oxidative stress and inflammation $[2,3]$. Nrf2deficient mice are more susceptible to oxidative stress [4] and inflammatory disorders including systemic inflammation [5], localized inflammation [6] and neuroinflammation [7]. Nrf2 works up-stream of HO-1, being a transcription factor sensitive to redox changes that lead to upregulation of several cytoprotective and detoxifying enzymes. Under normal conditions, $\mathrm{Nrf2}$ is retained in the cytoplasm in a 'silent' form by its repressor protein, Keap-1, which contains a subset of 27 highly reactive cysteine residues. Oxidative and environmental stimuli modify the cysteine residues of Keap-1, thus enabling translocation of Nrf2 to the nucleus where it binds to the antioxidant responsive element (ARE) located in the promoter region of detoxifying genes, including HO-1 [8]. Although Nrf2 and HO-1 are clearly linked in eliciting a protective action, 
the expression of HO-1 can also be modulated by others transcriptional regulators depending on the stimulus. In addition, HO-1 per se exerts a catalytic function by degrading heme to generate carbon monoxide (CO), iron and bilirubin as final by-products. These products account for many of the beneficial properties of HO-1 $[9,10]$, suggesting that pharmacological manipulation of the $\mathrm{Nrf} 2 / \mathrm{HO}-1$ axis could have important implications for limiting neuroinflammation. This notion is also supported by data obtained with CO-releasing molecules (CO-RMs), specific carriers of $\mathrm{CO}$ in biological systems that reduce the production of inflammatory markers in macrophages and microglia cells in culture $[11,12]$ and can decrease brain damage and inflammation in a rat model of hemorrhagic stroke [13].

Our interest in $\mathrm{Nrf} / \mathrm{HO}-1$ activators was initially motivated by results showing that curcumin, a natural polyphenolic compound, increased HO-1 levels in endothelial cells and elicited protection against $\mathrm{H}_{2} \mathrm{O}_{2}$-mediated oxidative damage in a HO-1-dependent manner [14]. We subsequently reported that HO-1 induction by curcumin was modulated by Nrf2 [15] and we studied other naturally-derived substances, such as chalcones and caffeic acid phenethyl esther, as promising inducers of the HO-1 pathway [16]. We also showed that the Michael acceptor functionality common to the chemical structure of many of these inducers is pivotal for promoting HO-1 up-regulation [17]. Because of their reliable response to inflammatory challenges, microglial cells in culture represent a good model to assess (brain) cellular adaptations following exposure to $\mathrm{Nrf2} / \mathrm{HO}-1$ activators.

In this study we performed an extensive literature search and selected 56 different compounds that were compared and evaluated for their ability to induce HO-1 and modulate inflammation in BV2 microglial cells. Using shRNA technology, we assessed the contribution of HO-1 and Nrf2 in mediating anti-inflammatory actions. We further concentrated on heme 
metabolism, showing that some compounds stimulate bilirubin production while others do not, despite their shared ability to up-regulate HO-1 expression. Our findings provide new insights on the chemical features and biochemical properties of small activators of $\mathrm{Nrf} 2 / \mathrm{HO}-1$ that are relevant for the control of inflammatory processes in microglia cells.

\section{Materials and Methods}

\subsection{Chemicals and reagents}

Supercurcumin (containing 95\% curcuminoids and piperine) was purchased from Life Extension, sildenafil from Sinova Inc (Bethesda, USA), 2,2'-dihydroxychalcone from Indofine Chemical Company Inc (Hillsborough, USA), hemin and tin protoporphyrin IX (SnPPIX) from Frontier Scientific (Logan, USA) and mouse interferon- $\gamma(\mathrm{INF}-\gamma)$ from ThermoFisher Scientific (Illkirch Cedex, France). All other chemicals and reagents were from Sigma unless otherwise stated.

\subsection{Cell culture}

BV2 mouse microglial cells were kindly donated by Professor Rosario Donato (University of Perugia) and Dr Adjanie Patabendige (Institute of Infection \& Global Health, Liverpool). Cells were cultured in either $75 \mathrm{~cm}^{2}$ flasks, 6-well or 24-well dishes containing RPMI medium supplemented with $10 \% \mathrm{FBS}, 4 \mathrm{mM}$ glutamine, $100 \mathrm{U} / \mathrm{ml}$ penicillin, $10 \mu \mathrm{g} / \mathrm{ml}$ streptomycin and grown in a $5 \% \mathrm{CO}_{2}$ atmosphere at $37^{\circ} \mathrm{C}$.

\subsection{Search for $\mathrm{Nrf2/HO}-1$ activators}


A literature search for compounds that induce HO-1 or Nrf2 was carried out using PubMed. The keywords used for the search were: a) HO-1 inducers and, b) Nrf2 inducers. The CAS databases were also searched using SciFinder to identify the chemical structure of various HO-1 inducers/Nrf2 activators reported in the literature. The SciFinder search used the same keywords as the PubMed search. From an original list of over 100 compounds identified in our preliminary search we created a compound library of 56 chemicals to be tested (Table 1); as some of the compounds were natural extracts or synthesized by the authors, the criteria for this selection were based on commercial availability of the substances and whether their chemical structure allowed for feasible structural modification.

\subsection{Immunoassay for $\mathrm{HO}-1$ protein expression}

A medium-throughput screening assay was utilized to determine the effect of the 56 compounds on HO-1 protein expression in BV2 cells. Confluent cells grown in 6-well dishes were treated for 6 or $24 \mathrm{~h}$ with increasing concentrations $(5,10$ or $20 \mu \mathrm{M})$ of each compound using also an internal positive control (10 $\mu \mathrm{M}$ hemin) for HO-1 protein expression in each experiment. DMSO, the solvent of most of the compounds assessed (see Table 1 for solubility information), was used in control cells. At the end of the incubation, cells were scraped and collected in PBS at $4{ }^{\circ} \mathrm{C}$. Samples were analyzed for HO-1 protein levels according to the protocol for the mouse ELISA (HO-1 (mouse) Immunoset, Vinci-Biochem).

\subsection{Cytotoxicity}

All 56 compounds were tested to evaluate their possible cytotoxic effects. BV2 cells were grown to confluence in 24-well dishes and exposed to 5, 10 or $20 \mu \mathrm{M}$ of each compound for $24 \mathrm{~h}$. Cells 
were also treated with a $1 \%$ Triton solution prepared in medium as a positive control (100\% cytotoxicity). Cell viability was determined using a lactate dehydrogenase (LDH) release assay (Cytotoxicity detection kit (LDH), Roche) according to manufacturers' instructions. Briefly, at the end of the incubation supernatants were collected and centrifuged at $250 \mathrm{x} g$ to remove any residual cell debris. The reaction mixture was added to the cell-free supernatant, and colour development was measured spectrophotometrically at $500 \mathrm{~nm}$.

\subsection{Heme oxygenase activity assay}

Cells cultured in $100 \mathrm{~mm}$ diameter petri dishes were collected $6 \mathrm{~h}$ after incubation with selected inducers $(20 \mu \mathrm{M})$ and assessed for heme oxygenase activity. The assay is based on the spectrophotometric determination of bilirubin as the final product of heme degradation by heme oxygenase [18]. Cell samples were incubated with the substrate hemin, NADPH, liver cytosol (a source of biliverdin reductase) and other co-factors to sustain heme oxygenase activity. The reaction was allowed to proceed for $1 \mathrm{~h}$ at $37^{\circ} \mathrm{C}$ in the dark and was terminated by addition of chloroform to extract the bilirubin produced. Bilirubin was measured spectrophotometrically as described before and calculated in picomoles bilirubin/mg protein/60 $\mathrm{min}$ [19].

\subsection{Determination of bilirubin released in the culture medium}

Confluent cells cultured in $60 \mathrm{~mm}$ diameter petri dishes were used for this assay. Cells were incubated with HO-1 inducers/Nrf2 activators for 6 or $24 \mathrm{~h}$ in the presence or absence of $10 \mu \mathrm{M}$ hemin in a final volume of $1 \mathrm{ml}$. At the end of the incubation, $0.5 \mathrm{ml}$ of culture supernatant was added to $250 \mathrm{mg}$ of $\mathrm{BaCl}_{2}$ and vortex-mixed thoroughly. Benzene was added to the mixture and tubes were vigorously vortex-mixed again. The benzene phase containing the extracted bilirubin 
was separated from the aqueous phase by centrifugation and bilirubin was measured spectrophotometrically [19,20]. Bilirubin was calculated in picomoles/ml of medium.

\subsection{Real-time PCR assay}

Confluent BV2 cells cultured in 6-well dishes were exposed for $4 \mathrm{~h}$ to certain compounds at 10 $\mu \mathrm{M}$ concentration. This time point was chosen based on preliminary experiments showing that maximal mRNA levels were achieved $4 \mathrm{~h}$ after incubation of cells with $10 \mu \mathrm{M}$ carnosol (data not shown). Total RNA was extracted from BV2 cells using the Qiagen RNeasy Mini Kit (Qiagen) and then reverse-transcribed using the Superscript First-Strand Synthesis System (Invitrogen) and Oligo(dT)20. Quantitative RT-PCR reactions were performed in triplicate on a 7900 real-time PCR detection system (Applied Biosystems) using Platinium SYBR Green qPCR SuperMix (Invitrogen). The following mouse primers were used in the reaction: HMOX1 forward primer:

5'-CCTCACTGGCAGGAAATCATC-3', HMOX1 reverse primer: 5'-

CTCGTGGAGACGCTTTACATA-3', GAPDH forward primer:

AAGAGAGGCCCTATCCCAAC, GAPDH reverse primer: GCAGCGAACTTTATTGATGG. PCR conditions were $50^{\circ} \mathrm{C}$ for $2 \mathrm{~min}, 95^{\circ} \mathrm{C}$ for $2 \mathrm{~min}, 45$ cycles at $95^{\circ} \mathrm{C}$ for $15 \mathrm{~s}$, and $60^{\circ} \mathrm{C}$ for 45 s, using GAPDH as the reference gene.

\subsection{Measurement of cellular heme content}

Heme content was determined in cells treated with HO-1 inducers in the presence or absence of succinylacetone $(1 \mathrm{mM})$, an inhibitor of heme biosynthesis and in medium containing 10 or $1 \%$ FBS. At the end of the incubation cells were washed twice with warm PBS, followed by addition of $1 \mathrm{ml}$ of formic acid to solubilize the cell layer as previously described [20]. The heme 
concentration in the formic acid solution was measured spectrophotometrically at $398 \mathrm{~nm}(\epsilon=$ $\left.1.56 \times 10^{5} \mathrm{M} / \mathrm{cm}\right)$. Heme uptake was expressed as picomole per well.

\subsection{Western blot analysis}

Expression of biliverdin reductase (BVR), delta-aminolevulinate synthase 1 (ALAS 1), HO-1 and Nrf2 were determined by western blot at different times after incubation with selected substances. In brief, an equal amount of proteins $(20 \mu \mathrm{g})$ for each sample was separated by SDSpolyacrylamide gel electrophoresis and transferred to nitrocellulose membranes; the nonspecific binding of antibodies then was blocked with 5\% nonfat dried milk and $0.1 \%$ Tween in TBS. Membranes were then probed overnight with a rabbit polyclonal antibodies against HO-1 or biliverdin reductase (Stressgen, 1:1000 dilution), Nrf2 (Santa Cruz Biotechnology, Inc., 1:1000 dilution) and ALAS 1 (ABCAM, 1:1000 dilution). After incubation with horseradish-peroxidaseconjugated secondary antibody (Cell Signaling Technology, 1:5000 dilution), bands were visualized using Pierce ECL revelation kit.

\subsection{Silencing of HO-1 or Nrf2 using shRNA}

Small hairpin RNA specific for mouse HO-1 or Nrf2 and a negative control (sc-108080) obtained from Santa Cruz (Santa Cruz Biotechnology) were used to infect BV2 cells. Lentiviral particles were added to the cell culture in a 6-well dish and cells were incubated overnight. The following day the medium was replaced with medium containing puromycin for selection of stably transfected colonies following manufactures' instructions.

\subsection{Nitrite assay and detection of TNF $\alpha$ and PGE2 after inflammatory challenges}


BV2 cells grown in 24-well dishes were treated for $24 \mathrm{~h}$ with lipopolysaccharide (LPS, 0.5 $\mu \mathrm{g} / \mathrm{ml})$ or INF- $\gamma(15 \mathrm{ng} / \mathrm{ml})$ in the presence or absence of test compounds $(5,10$ or $20 \mu \mathrm{M})$. In some experiments the heme oxygenase activity inhibitor SnPPIX $(10 \mu \mathrm{M})$ or bilirubin $(0.5$ to 10 $\mu \mathrm{M})$ were included. Cells in which HO-1 or Nrf2 were silenced by shRNA were also used. At the end of the incubation the supernatant was collected for measurements of nitrite levels using the Griess method [11] while TNF $\alpha$ and PGE2 levels were assessed by ELISA following manufacturers' instructions (Enzo Life Sciences, Villeurbanne, France).

\subsection{Data analysis}

Statistical analysis was performed using Graph Pad Prism. One-way ANOVA combined with the Bonferroni or Tukey's multiple comparison tests and the T-test were applied. Differences were considered to be significant at $P<0.05$. The EC50 was calculated for positive compounds used at 5, 10 and $20 \mu \mathrm{M}$, thus representing an approximate rather than a definite value since a full dose response curve to each of the compound used was not performed in the present study.

\section{Results}

\subsection{Screening of 56 small molecules activators for $\mathrm{HO}-1$ expression and cytotoxicity}

The initial literature search identified more than 100 compounds, including in some cases derivatives, which induce or activate HO-1 and/or Nrf2. These compounds had been studied by various authors using different cell types, different cell culture conditions and different experimental protocols. Thus, one aim of our study was to evaluate and standardize the effect of these small molecules within the same cellular system and under the same experimental conditions. Some of the compounds were synthesized by the authors of the article, others were 
commercially available (e.g., oleanolic acid derivatives, carnosol, epigallocatechin, lansoprazole, flunarizine), while many others, such as isoegomaketone, sauchinone, or sulfuretin, were extracted from natural sources. From this initial list, 56 priority compounds were chosen to be tested for HO- 1 expression after 6 and $24 \mathrm{~h}$ incubation at three different concentrations $(5,10$ and $20 \mu \mathrm{M})$ in microglia cells. Their effect on cell viability was also assessed. We did not test concentrations higher than $20 \mu \mathrm{M}$ since we observed in previous experiments that some HO-1 inducers start to exhibit significant cytotoxicity around 25-30 $\mu \mathrm{M}$ [14]. In addition, the usefulness of compounds which can increase HO-1 at concentrations higher than $20 \mu \mathrm{M}$ might be limited when there exists already molecules that are effective in the lower micromolar range. This is especially true if these compounds exhibit other beneficial properties, such as anti-inflammatory action, that are manifested at low micromolar doses. We note that the bioavailability of some naturally-derived compounds is limited and therefore we are unable to determine if the concentrations used in our study will be achieved in the brain after oral intake. Table 1 summarizes the results of the screening, reporting the name of the compounds, their capacity to induce HO-1 at 6 and $24 \mathrm{~h}$, their solubility as well as their cytotoxic profile. From these initial experiments it is evident that many of the compounds (35) did not increase HO-1 in BV2 at the concentrations tested. These included, among others, acethaminophen, capsaicin, eriodictyol, probucol and sildenafil, which have been shown to augment HO-1 in other cell types [21-25]. We also observed that 10 compounds caused, in parallel with HO-1 induction, a release of LDH $\geq 15 \%$, underlying some intrinsic cytotoxic properties. We set $15 \%$ LDH release as a reasonable limit for cell toxicity since the HO-1-inducing capacity of compounds should be balanced against their negative effects on cells. It is important to note that 7 out of the 10 more cytotoxic substances maintained up-regulation of HO- 1 up to $24 \mathrm{~h}$, suggesting that HO-1 expression is 
prolonged to counteract cell damage. In contrast, compounds such as carnosol (CA), dimethyl fumarate (DMF), supercurcumin (SC) and cobalt protoporphyrin IX (CoPP) stimulated HO-1 at 6 and $24 \mathrm{~h}$ with minor effects on cell viability (see also Table 2). Altogether, the data from this initial screening indicate that out of 56 compounds, the natural derivatives carnosic acid (CAA), CA, DMF and SC together with the porphyrin CoPP exhibit the best HO-1 inducing/cytotoxicity profile in BV2 cells. We selected from the first set of results 10 positive leads (including hemin as our internal positive, see Table 2) and 5 negatives. Apart from their inability to promote HO-1 induction, the negatives eriodictyol (E), probucol (PRO), resveratrol (R) and sildenafil (SI) did not affect cell viability (Table 3). The only exception was plumbagin (PLU), which was highly toxic even at $5 \mu \mathrm{M}$. Quantitative real time PCR confirmed induction of the HMOX1 gene for $\mathrm{H}$, DMF, CA, SC (Fig. 2C) after $4 \mathrm{~h}$ exposure. The negatives PRO and RES also slightly increased HMOX1 gene although this effect did not translate into a significant increase in HO-1 protein expression (measured at $6 \mathrm{~h}$ after exposure). Indeed, no significant correlation was found between increases in mRNA and protein levels after incubation with $10 \mu \mathrm{M} \mathrm{H}, \mathrm{DMF}, \mathrm{CA}, \mathrm{SC}$, PRO and RES $\left(r^{2}=0.16\right.$, $\mathrm{P}$ value $\left.=0.32\right)$, suggesting that augmented HO- 1 expression by these compounds is not driven exclusively by an increase in mRNA.

\subsection{Endogenous production of bilirubin by lead compounds}

Since many of the protective actions mediated by HO-1 are dependent on its final degradation products bilirubin and $\mathrm{CO}$ (and ferritin as a scavenging protein for iron), we wondered whether increased HO-1 expression by positive compounds was associated with a rise in HO-1-derived products. We measured bilirubin, which accumulates in the cell supernatant and can be detected spectrophotometrically. A rise in bilirubin also indicates a concomitant production of CO. Fig. 
1A shows that of the 10 positives tested at $20 \mu \mathrm{M}, \mathrm{CoPP}, \mathrm{CA}, \mathrm{SC}, \mathrm{CAA}$ and tertbutylhydroquinone (tBH) stimulated an increase in cellular bilirubin production at $6 \mathrm{~h}$ and/or 24 h. The internal positive control hemin, the putative inducer and substrate of heme oxygenase activity, was used at $10 \mu \mathrm{M}$ concentration and promoted the expected accumulation of bilirubin [20] in the cell culture media. These results differentiate HO-1 inducers between those capable of stimulating consumption of heme by heme oxygenase and those which do not, despite their shared ability to induce high HO-1 expression. To ensure that a functional heme oxygenase was expressed following activation by the compounds, we repeated the same experiments by incubating cells with the selected substances in the presence of $10 \mu \mathrm{M}$ hemin. Thus, when hemin was provided exogenously as the substrate for heme oxygenase activity, bilirubin increased both at 6 (Fig. $1 B$ ) and $24 \mathrm{~h}$ (Fig. 1C) with all substances examined. In the case of CoPP, CAA, 2,2dihydroxychalcone (DHC) and tBH this increase was significantly higher than with either hemin or the substance alone, suggesting an additive or synergistic action.

\subsection{What is the source of heme utilized by heme oxygenase following induction by certain}

\section{inducers?}

The data reported above describe that certain inducers concomitantly stimulated HO-1 expression and bilirubin production in BV2 while others elicited only HO-1 induction. To better understand this phenomenon we concentrated on SC and CA and first examined if inhibition of heme biosynthesis by succinylacetone affected their capacity to increase bilirubin. For these experiments succinylacetone was pre-incubated for $16 \mathrm{~h}$ and maintained in the culture medium also during the entire exposure of cells to HO-1 inducers. In addition, we cultured cells in medium containing either 10 or $1 \%$ FBS, since this medium component actually contains 
detectable amounts of hemoglobin and could thus be an external source of heme for use by cells. We used $10 \mu \mathrm{M}$ hemin as positive control. Fig. 2A shows that bilirubin was produced in equal amounts when cells were exposed to hemin in the presence or absence of succinylacetone. This was expected, as hemin should enter the cell, up-regulate HO-1 and be degraded by the enzyme regardless and independently of heme synthesis inhibition. This effect was evident in medium containing either $10 \%$ or $1 \%$ FBS but with an important difference: the amount of bilirubin produced by cells incubated in $1 \%$ FBS medium was much higher compared with that measured in cells cultured in $10 \%$ FBS-containing medium. Concerning SC and CA, the results of the experiments carried out in 10\% FBS do not clearly support that heme resulting from de novo synthesis was used as a substrate for production of bilirubin by SC or CA, although succinylacetone showed a non-significant tendency to decrease bilirubin levels with SC (Fig. 2A, left panel). On the contrary, our data point to FBS as the major source of heme for the production of bilirubin, since this increase was completely absent in BV2 cells incubated with SC or CA in 1\% FBS (Fig. 2A, right panel). The measurement of cellular heme content indicated that treatment of cells with hemin, but not with SC or CA, increased heme levels and that this effect was much more pronounced in cells cultured in 1\% FBS than 10\% FBS (Fig. 2B). Succinylacetone caused a slight, non significant decrease in heme content (Fig. 2B) at $1 \mathrm{mM}$; we decided against the use of this compound at higher concentrations to avoid possible cytotoxicity [26].

3.4. Effect of small activators on expression of heme synthesis and degradation proteins (ALAS 1 and $B V R)$ 
The pathways involving heme metabolism encompass multiple cytosolic and mitochondrial enzymes that regulate various passages of heme synthesis and degradation. The fact that some HO-1 inducers stimulate bilirubin production, while others do not, was intriguing and prompted us to explore whether pathways working alongside HO-1 in the heme metabolic reactions were also affected by the inducers. To confirm the results obtained with succinylacetone, we verified the expression levels of ALAS 1, the rate-limiting enzyme in heme biosynthesis [27] following 6 $\mathrm{h}$ incubation with selected substances. As shown in Fig. 3A, the expression of ALAS 1 tended to decrease in BV2 cells exposed to the positives SC, CA and DMF and the 2 negatives PRO and R but this difference was not statistically different $(\mathrm{P}$ value $=0.1352)$. In addition, we aimed to determine whether inducers changed the levels of BVR in parallel to HO-1. BVR is an inducible protein that reduces biliverdin to bilirubin but also exhibits cytoprotective and anti-inflammatory properties independently of HO-1 [28]. However, BVR expression levels remained virtually unchanged after $6 \mathrm{~h}$ exposure to SC, CA, DMF, PRO and R (Fig 3 B). We also tested if Nacetylcysteine (NAC, $1 \mathrm{mM}$ ), an antioxidant and a precursor of glutathione synthesis, influenced HO-1 induction by the inducers. Fig 3C shows that pre-incubation of BV2 with NAC diminished the increased heme oxygenase activity elicited by all the inducers evaluated (including hemin), suggesting a contribution of thiol/redox reactions in the mechanisms underlying HO-1 induction by Nrf2/HO-1 small molecule activators.

\subsection{Small molecules differentially modulate inflammatory markers in BV2 cells challenged with}

\section{LPS or INF- $\gamma$}

The ability of the selected compounds to modulate LPS or INF $\gamma$-induced inflammation in BV2 cells was assessed. We were keen to distinguish the response to LPS, which directly activates 
TLR4 receptors and stimulates acute inflammation, from that of INF- $\gamma$, a cytokine derived from peripheral immune cells that may better mimic a low level of chronic inflammation. Nitrite, an index of nitric oxide production, was measured in the cell supernatant $24 \mathrm{~h}$ after co-incubation of small activators with INF- $\gamma$ or LPS. This protocol differs from our previously published works [29], in which we pre-incubated inflammatory cells with HO-1 inducers at least $6 \mathrm{~h}$ prior to LPS challenge, thus ensuring high expression of HO-1 before the application of the inflammatory stimulus. Fig. 4 shows that most positives used at $20 \mu \mathrm{M}$ concentration decrease nitrite in cells challenged with INF- $\gamma$; tBH, CA, CoPP, ISL, SC and $\mathrm{S}$ were all effective at the highest concentration while $\mathrm{CA}, \mathrm{CoPP}$ and $\mathrm{S}$ elicited marked decreases also at 5 and $10 \mu \mathrm{M}$. Inhibiting heme oxygenase activity with SnPPIX partially reversed the reduction in nitrite caused by $\mathrm{tBH}$, CA, DMF, S and SC (Supplementary Fig. S1). We also measured INF- $\gamma$-induced nitrite levels in the presence of the negative compounds since they may exert anti-inflammatory properties independent of HO-1. In these experiments we observed that E and PLU markedly diminished nitrite production, although this effect was probably due to high cytotoxicity in the case of PLU (Fig. 5). When inflammation was triggered by LPS we observed a very good concentrationdependent inhibition of nitrite production by all positives (Fig. 6), with CA, CoPP, S and SC being the most potent. However, SnPPIX did not seem very effective in reversing this effect with all the compounds tested (Supplementary Fig. S2). As observed with INF- $\gamma$, some negatives, such as E, R and PLU could also decrease nitrite production stimulated by LPS (Fig. 7). We found that the combination of sulphoraphane with LPS or INF- $\gamma$ caused increased microglia toxicity in vitro (data not shown). These results confirm that HO-1 inducers can attenuate inflammatory responses in microglia cells and suggest that the contribution of the HO-1 pathway to this effect is stronger for INF- $\gamma$-stimulated inflammation than for LPS. In addition, these data highlight that some 
negatives still exert anti-inflammatory action which likely involves modulation of HO-1independent mechanisms.

\subsection{Correlation between bilirubin production induced by small activators and nitrite levels}

As already described, HO-1-derived products mediate many of the defensive properties of heme oxygenase. Therefore, we correlated the levels of bilirubin produced by positives versus the amount of nitrite measured after exposure of cells to INF- $\gamma$ or LPS in the presence of the compounds. We excluded compound S from this analysis since the LDH release in cells exposed to $20 \mu \mathrm{M}$ of the compound alone was $15.8 \%$ but markedly increased to $>70 \%$ when exposed simultaneously with INF- $\gamma$ or LPS (data not shown). Thus, the decrease in nitrite in the presence of compound $\mathrm{S}$ is most likely a consequence of substantial cell toxicity rather than an effect mediated by HO-1 induction. The results of this correlation are very interesting as they underline an inverse, significant relationship between bilirubin and nitrite production in the case of cells challenged with INF- $\gamma$ (Fig. 8A) but a weaker and non-significant relationship for LPS-treated cells (Fig. 8B). These results suggest that HO-1 induction and its enzymatic reaction are linked to the anti-inflammatory action of the positive compounds tested. The data are also in line with those obtained with the heme oxygenase inhibitor, highlighting a stronger reliance of positives on HO-1 in counteracting inflammation caused by INF- $\gamma$ than LPS, at least concerning the nitrite response.

3.7. Contribution of HO-1 and Nrf2 in the modulation of inflammatory markers by small molecule activators 
To assess whether the Nrf2/HO-1 contributed to the inflammatory-modulating properties of small molecule activators, we treated cells with shRNA to knock down HO-1 or Nrf2 and examined the generation of nitrite, prostaglandin $\mathrm{E}_{2}\left(\mathrm{PGE}_{2}\right)$ and tumor necrosis factor- $\alpha$ (TNF- $\alpha$ ) induced by INF- $\gamma$ or LPS. Control cells were treated with scrambled shRNA and all compounds were used at $10 \mu \mathrm{M}$ concentration. Fig. 9 illustrates the data obtained in INF- $\gamma$-challenged BV2. As shown in Fig. 9A, nitrite production increased in control cells incubated with INF- $\gamma$. Silencing of HO-1 or Nrf2 further increased nitrite levels and this effect was more pronounced in Nrf2-lacking cells. Nitrite was substantially decreased by tBH, CA and CoPP and, while down-regulation of HO-1 or Nrf2 significantly reversed this outcome in the case of tBH and CA, nitrite levels remained consistently low in scrambled, HO-1 or Nrf2 shRNA-treated cells in the presence of CoPP. The nitrite profile observed with the positive DMF or the negatives PRO and R did not differ from that of cells exposed to INF- $\gamma$ alone. Measuring $\mathrm{PGE}_{2}$ provided an interesting and unexpected picture as this inflammatory mediator was increased in scrambled-infected cells challenged with INF- $\gamma$ but was reduced to control levels in HO-1 or Nrf2-silenced cells (Fig. 9B). Levels of $\mathrm{PGE}_{2}$ were similar to control in scramble, HO-1 or Nrf2 shRNA-treated cells in the presence of INF- $\gamma$ and all the positives or negatives tested. Similarly, the production of TNF- $\alpha$ was increased by INF- $\gamma$ in scrambled-infected cells (Fig. 9C) but was undetectable in HO-1 or Nrf2-silenced cells. Most of the positives and the two negatives tested decreased to some extent TNF- $\alpha$ levels stimulated by INF- $\gamma$ in scrambled control cells, however, knocking down HO-1 or Nrf2 did not restore TNF- $\alpha$ production. The only exception to this trend was observed with the positive DMF. In fact, TNF- $\alpha$ was not inhibited in scrambled control cells co-incubated with INF- $\gamma$ and DMF, was markedly reduced in HO-1 silenced cells but its levels were re-established in Nrf2-silenced BV2. These data collected in INF- $\gamma$-challenged microglia highlight the following: 1) Nrf2/HO-1 
small molecule activators modulate the INF- $\gamma$-mediated inflammatory response; 2) HO-1 and Nrf2 appear implicated in mediating the effect of small molecule activators on nitric oxide production but not for $\mathrm{PGE}_{2}$ or TNF- $\alpha$ and 3) the two negative compounds assessed exhibit independent inflammation modulating properties.

Challenge of BV2 with LPS generated a somewhat diverse outcome (Fig. 10). For example, the positives tested reduced nitrite production but to a smaller extent compared to the results observed with INF- $\gamma$ (Fig. 10A). The most effective compound in this set of experiments was CA and silencing of Nrf2, rather than HO-1, more strongly reversed this effect also in the case of the negatives PRO and R. Furthermore, and in line with the data acquired from INF- $\gamma$-treated cells, LPS stimulated a substantial $\mathrm{PGE}_{2}$ production that was decreased in Nrf2-silenced cells but even more when HO-1 was down-regulated (Fig. 10B). The presence of tBH, CA and DMF markedly diminished $\mathrm{PGE}_{2}$ in scrambled, $\mathrm{HO}-1$ or Nrf2-treated cells and similar trends were observed with the negatives $\mathrm{R}$ and to a lesser extent PRO. In contrast to the INF- $\gamma$ data, CoPP did not alter the response compared to LPS alone. The final measurement of TNF- $\alpha$ indicated that this marker increased in control scramble cells stimulated with LPS (Fig. 10C). Importantly, this increase was substantially augmented when HO-1 or Nrf2 were silenced. Apart from CoPP, the positives tBH, CA, DMF and the negative R diminished TNF- $\alpha$ induced by LPS in control cells and a tendency to reverse this effect was observed mostly in cells silenced for HO-1. Thus, we observe that following LPS stimulation small molecule activators differentially affect the markers of inflammation we chose to examine. The results also suggest that HO-1 and/or Nrf2 contribute to the effect mediated by the compounds either by counteracting the production of certain markers (nitrite and TNF- $\alpha$ ) or by promoting their generation $\left(\mathrm{PGE}_{2}\right)$. Altogether, the data also emphasize 
important differences between INF- $\gamma$ and LPS-induced inflammation and the roles of HO-1 and Nrf2 in the cellular response to these stimuli. 


\section{Discussion}

Manipulation of the $\mathrm{Nrf} 2 / \mathrm{HO}-1$ pathways has been shown experimentally to protect against a variety of conditions characterized by oxidative damage and inflammation $[3,30]$ and targeting Nrf2/HO-1 with appropriate pharmacologically-active compounds may help to reduce the progression of chronic diseases, including neurodegeneration. It is well established that microglia activation following inflammatory challenge modulates neuroprotection [1,31], suggesting that controlling microglia activation by pharmacological approaches may be one useful step towards the reduction of neuronal damage.

We report in this study the results of a screening test of 56 compounds on the up-regulation of the cytoprotective protein $\mathrm{HO}-1$ in BV2 microglia cells in vitro. The compounds were selected over more than 100 found during a literature search where $\mathrm{HO}-1$ and/or Nrf2 were the keywords used. We compared their HO-1 stimulating capacity with their effect on cell viability. We selected 10 positives (including hemin) and 5 negatives to further examine their effect on heme metabolism and modulation of the inflammatory response. This investigation identifies carnosic acid (CAA), carnosol (CA), cobalt protoporphyrin IX (CoPP), dimethyl fumarate (DMF) and supercurcumin (SC) as the compounds eliciting the best HO-1 induction and low cytotoxicity. We also found that the compounds differentiate between those capable of promoting the generation of HO-1-derived products $(\mathrm{CA}, \mathrm{CoPP}, \mathrm{SC})$ and others that do not, indicating that $\mathrm{HO}$ 1 induction by specific molecules may be independent from its heme-degradation function. When assessed for their inflammatory-modulating properties, we observed that the positives reduced more strongly inflammatory mediators stimulated by INF- $\gamma$ than LPS. In addition, the use of shRNA to silence HO-1 or Nrf2 showed that the two pathways contribute to the effect of the compounds to counteract nitric oxide production but not $\mathrm{PGE}_{2}$ or TNF- $\alpha$ in cells challenged with 
INF- $\gamma$, while HO-1 and Nrf2 were substantially implicated in modulating all the inflammatory markers examined after LPS stimulation. These data highlight the pleiotropic activities of the compounds examined in this study, the different response to LPS and INF- $\gamma$-mediated inflammation and the importance of comparing compounds with supposedly similar biological activity within the same cellular system.

The majority of the positive compounds studied in this report possess electrophilic features, which are necessary for activation of Nrf2 and the induction of Nrf2-dependent genes such as HO-1. However, despite investigating many substances, the best profile for HO-1 induction/low cell toxicity was mostly achieved by some of the original compounds first identified many years ago as HO-1 inducers (i.e. curcumin) [14] and some later identified electrophilic compounds (carnosol, chalcones) [32-34], suggesting that their chemical structure is perhaps unique and 'evolutionarily' selected to specifically activate the Nrf2/HO-1 pathway. This chemical feature is the base for the development of synthetic triterpenoids, a group of Nrf2 activators recently demonstrated to reduce neurotoxicity, oxidative stress and inflammation in a mouse model of Parkinson's disease [35]. However, the results of a recent clinical trial using a similar compound for advanced chronic kidney disease suggests that a better understanding of the biological behavior of these new molecules and a fine-tuning of their pharmacological vs. cytotoxic properties is required to fully exploit their therapeutic potential [36].

Apart from hemin, the only HO-1 inducer which is not electrophilic is CoPP. This compound can act as substrate for heme oxygenase activity, although it is less effective than the natural substrate heme [37]. However, CoPP up-regulated Nrf2 in human hepatocytes via posttranscriptional site(s) of action [38] and protected cardiac stem cells from apoptosis in a Nrf2dependent manner [39], indicating that electrophilicity might not be the only chemical property 
required for $\mathrm{Nrf} 2 / \mathrm{HO}-1$ activation. Our data showing that $\mathrm{NAC}$ reduced the increase in heme oxygenase by most inducers point to oxidative stress/thiol regulation as important components for this induction. Since mouse, rat and human primary microglia have been shown to overexpress HO-1 and activate Nrf2 following various stimuli, we suggest that the Nrf2/HO-1 activators investigated in the present study will exert similar effects also in primary cells.

Our results also show that not all HO-1 inducers stimulate bilirubin production. It was intriguing to find that for those compounds that induced HO-1 (CA and SC), the source of heme was external, i.e. the fetal bovine serum with its residual haemoglobin content $(18.5 \mathrm{mg} / 100 \mathrm{ml}$ according to the certificate of analysis of the FBS used in our cell culture work). This idea is supported by our data indicating that: 1) no bilirubin production was observed when cells were incubated in medium containing only $1 \%$ serums; 2) pharmacological inhibition of heme biosynthesis did not affect bilirubin release in the supernatant in the presence of CA and SC and 3) protein expression of other heme metabolism pathways directly related to heme synthesis and degradation (ALAS 1 synthase or BVR) was not changed by CA and SC. The use of exogenous heme sources was unlikely to depend from cytotoxicity since CA and SC were among the least toxic compounds at the concentrations used. A recent article by Zelenka et al. has measured bilirubin production both intracellularly and in the culture medium by HPLC, showing that bilirubin is increased in HepG2 cells following HO-1 induction by sodium arsenite and thus confirming the ability of cells in culture to generate detectable levels of bilirubin after exposure to the appropriate stimuli [40].

Our approach helped us to compare the molecules in terms of their anti-inflammatory activity, in order to select the ones that best modulate inflammation in microglia. Positive compounds, and to an extent also some negatives (E for INF- $\gamma$ and R for LPS), decreased nitric 
oxide generation induced by INF- $\gamma$ or LPS and inhibition of heme oxygenase activity partially reversed this effect. The degree of nitrite reduction was different for each small molecule and we noted a stronger contribution of the HO-1 pathway in cells exposed to INF- $\gamma$ - than to LPS. Indeed, the correlation analysis showed a significant inverse correlation between nitrite levels and bilirubin production elicited by the compounds in INF- $\gamma$-treated cells. This pattern is interesting and suggests that higher bilirubin levels stimulated by some substances results in lower nitrite released by microglia following INF- $\gamma$. However, bilirubin applied exogenously ( 0.5 to $10 \mu \mathrm{M})$ did not change nitrite levels (data not shown). Thus, we suggest that this effect may be due to $\mathrm{CO}$, produced simultaneously to bilirubin during heme degradation by heme oxygenase, as shown previously for CO-releasing molecules in murine macrophages and BV2 cells [12,41].

In general, the inflammatory response promoted by LPS was more robust than with INF- $\gamma$; possibly emphasizing a different microglia response to acute (LPS) versus chronic low level inflammation (INF- $\gamma$ ). In line with this idea, Orozco et al have shown in a large study involving 92 mouse strains that changes in gene expression are more pronounced in primary macrophages exposed to LPS than to oxidized phospholipids, which also leads to chronic inflammation [42]. Interestingly, the study revealed that HO-1 was markedly regulated in response to oxidized phospholipids but not to LPS. Our data using cells silenced for HO-1 or Nrf2 also highlight intriguing observations. First, we noted that in the absence of positives or negatives, silencing HO-1 or Nrf2 modulated the production of inflammatory markers induced by INF- $\gamma$ or LPS; in particular, nitrite levels were enhanced while $\mathrm{PGE}_{2}$ was decreased. Conversely, lack of HO-1 or Nrf2 abolished TNF- $\alpha$ release in cells challenged with INF- $\gamma$ but augmented it in the case of LPS. Thus, HO-1 and Nrf2 counteract nitric oxide production but support the generation of $\mathrm{PGE}_{2}$. This inflammatory lipid mediator deriving from cyclooxygenase- 2 exhibits both pro- and anti- 
inflammatory properties and may have different roles depending on the condition examined. Second, knocking down HO-1 or Nrf2 influenced more nitrite production by the compounds in cells challenged with INF- $\gamma$. The compounds (including the negatives PRO and R) completely blocked $\mathrm{PGE}_{2}$ production by INF- $\gamma$ and silencing $\mathrm{HO}-1$ or $\mathrm{Nrf} 2$ did not restore $\mathrm{PGE}_{2}$ levels. This effect was similar but less pronounced in LPS-stimulated microglia. Our findings are in line with a recent report showing that $\mathrm{R}$ inhibited LPS-mediated stimulation of $\mathrm{PGE}_{2}$ by $\mathrm{BV} 2$ via the mammalian target of rapamycin signalling pathway [43]. They also demonstrate that natural based molecules can exert similar anti-inflammatory activity by modulating cellular mechanisms alternative or parallel to $\mathrm{HO}-1$ and Nrf2. Third, the inflammatory-regulating properties of the compounds were better differentiated in LPS-treated cells. For example, CA was the most effective regulator of the response while tBH or DMF had less influence on nitrite production but affected more $\mathrm{PGE}_{2}$ and TNF- $\alpha$ levels. Although the bioavailability of these compounds may be limited in vivo, published findings in animals show beneficial effects of sulforaphane, curcumin and other Nrf2 activators in brain inflammation [3,44].

In conclusion, we have identified potent activators of the Nrf2/HO-1 pathways (i.e. carnosol, cobalt protoporphyrin, dimelthyl fumarate, supercurcumin) capable of modulating inflammation in BV2 microglia, suggesting a complex role of these compounds in controlling the production of inflammatory mediators. Our results also point to heme metabolism as an additional factor affecting the actions of these compounds, indicating that HO-1 induction in the absence of heme degradation may limit the cytoprotective potential of this enzyme. This screening approach suggests that modification of selected chemical scaffolds may be promising for the synthesis of new molecules to combat brain inflammation and neurodegeneration, as recently shown by Chen 
Q et al. with the synthesis of potent, orally active curcumin derivatives that prevented cognitive decline in a mouse model of Alzheimer's disease [45].

\section{Acknowledgements}

A preliminary part of this work was conducted when R.F., S.K.B., T.P., L.E.B.C. and R.M. were working at the Department of Drug Discovery and Development, Italian Institute of Technology, Genova, Italy. We thank AREMCAR Foundation for financial support and the International $\mathrm{PhD}$ Program in Neuropharmacology, Medical School, University of Catania. This work was supported in part by a grant from the Agence Nationale de la Recherche (MITO-CO) to Dr. Roberto Motterlini.

\section{References}

[1] Skaper SD, Giusti P, Facci L. Microglia and mast cells: two tracks on the road to neuroinflammation. FASEB J 2012;26:3103-3117.

[2] Jazwa A and Cuadrado A. Targeting heme oxygenase-1 for neuroprotection and neuroinflammation in neurodegenerative diseases. Curr Drug Targets 2010;11:1517-1531.

[3] Innamorato NG, Rojo AI, Garcia-Yague AJ, Yamamoto M, de Ceballos ML, Cuadrado A. The transcription factor $\mathrm{Nrf2}$ is a therapeutic target against brain inflammation. J Immunol 2008;181:680-689.

[4] Yoh K, Hirayama A, Ishizaki K, Yamada A, Takeuchi M, Yamagishi S, Morito N, Nakano T, Ojima M, Shimohata H, Itoh K, Takahashi S, Yamamoto M. Hyperglycemia induces oxidative and nitrosative stress and increases renal functional impairment in Nrf2deficient mice. Genes Cells 2008;13:1159-1170. 
[5] Thimmulappa RK, Lee H, Rangasamy T, Reddy SP, Yamamoto M, Kensler TW, Biswal S. Nrf2 is a critical regulator of the innate immune response and survival during experimental sepsis. J Clin Invest 2006;116:984-995.

[6] Braun S, Hanselmann C, Gassmann MG, Auf dem KU, Born-Berclaz C, Chan K, Kan YW, Werner S. Nrf2 transcription factor, a novel target of keratinocyte growth factor action which regulates gene expression and inflammation in the healing skin wound. Mol Cell Biol 2002;22:5492-5505.

[7] Kitamuro T, Takahashi K, Ogawa K, Udono-Fujimori R, Takeda K, Furuyama K, Nakayama M, Sun J, Fujita H, Hida W, Hattori T, Shirato K, Igarashi K, Shibahara S. Bach1 functions as a hypoxia-inducible repressor for the heme oxygenase-1 gene in human cells. J Biol Chem 2003;278:9125-9133.

[8] Baird L and Dinkova-Kostova AT. The cytoprotective role of the Keap1-Nrf2 pathway. Arch Toxicol 2011;85:241-272.

[9] Motterlini R and Otterbein LE. The therapeutic potential of carbon monoxide. Nat Rev Drug Discov 2010;9:728-743.

[10] Bulmer AC, Verkade HJ, Wagner KH. Bilirubin and beyond: A review of lipid status in Gilbert's syndrome and its relevance to cardiovascular disease protection. Prog Lipid Res 2012;52:193-205.

[11] Sawle P, Foresti R, Mann BE, Johnson TR, Green CJ, Motterlini R. Carbon monoxidereleasing molecules (CO-RMs) attenuate the inflammatory response elicited by lipopolysaccharide in RAW264.7 murine macrophages. Br J Pharmacol 2005;145:800810.

[12] Bani-Hani MG, Greenstein D, Mann BE, Green CJ, Motterlini R. Modulation of thrombin-induced neuroinflammation in BV-2 microglia by a carbon monoxide-releasing molecule (CORM-3). J Pharmacol Exp Ther 2006;318:1315-1322.

[13] Yabluchanskiy A, Sawle P, Homer-Vanniasinkam S, Green CJ, Foresti R, Motterlini R. CORM-3, a carbon monoxide-releasing molecule, alters the inflammatory response and 
reduces brain damage in a rat model of hemorrhagic stroke. Crit Care Med 2012;40:544552.

[14] Motterlini R, Foresti R, Bassi R, Green CJ. Curcumin, an antioxidant and antiinflammatory agent, induces heme oxygenase- 1 and protects endothelial cells against oxidative stress. Free Rad Biol Med 2000;28:1303-1312.

[15] Balogun E, Hoque M, Gong P, Killeen E, Green CJ, Foresti R, Alam J, Motterlini R. Curcumin activates the heme oxygenase-1 gene via regulation of Nrf2 and the antioxidant responsive element. Biochem J 2003;371:887-895.

[16] Scapagnini G, Foresti R, Calabrese V, Stella AM, Green CJ, Motterlini R. Caffeic acid phenethyl ester and curcumin: a novel class of heme oxygenase-1 inducers. Mol Pharmacol 2002;61:554-561.

[17] Sawle P, Moulton BE, Jarzykowska M, Green CJ, Foresti R, Fairlamb IJ, Motterlini R. Structure-Activity Relationships of Methoxychalcones as Inducers of Heme Oxygenase-1. Chem Res Toxicol 2008;21:1484-1494.

[18] Vesely MJJ, Exon DJ, Clark JE, Foresti R, Green CJ, Motterlini R. Heme oxygenase-1 induction in skeletal muscle cells: hemin and sodium nitroprusside are regulators in vitro. Am J Physiol 1998;275:C1087-C1094.

[19] Clark JE, Foresti R, Green CJ, Motterlini R. Dynamics of haem oxygenase-1 expression and bilirubin production in cellular protection against oxidative stress. Biochem $\mathrm{J}$ 2000;348:615-619.

[20] Foresti R and Motterlini R. The heme oxygenase pathway and its interaction with nitric oxide in the control of cellular homeostasis. Free Rad Res 1999;31:459-475.

[21] Goldring CE, Kitteringham NR, Elsby R, Randle LE, Clement YN, Williams DP, McMahon M, Hayes JD, Itoh K, Yamamoto M, Park BK. Activation of hepatic Nrf2 in vivo by acetaminophen in CD-1 mice. Hepatology 2004;39:1267-1276. 
[22] Deng YM, Wu BJ, Witting PK, Stocker R. Probucol protects against smooth muscle cell proliferation by upregulating heme oxygenase-1. Circulation 2004;110:1855-1860.

[23] Vidavalur R, Penumathsa SV, Zhan L, Thirunavukkarasu M, Maulik N. Sildenafil induces angiogenic response in human coronary arteriolar endothelial cells through the expression of thioredoxin, hemeoxygenase and vascular endothelial growth factor. Vascul Pharmacol 2006;45:91-95.

[24] Joung EJ, Li MH, Lee HG, Somparn N, Jung YS, Na HK, Kim SH, Cha YN, Surh YJ. Capsaicin induces heme oxygenase-1 expression in HepG2 cells via activation of PI3KNrf2 signaling: NAD(P)H:quinone oxidoreductase as a potential target. Antioxid Redox Signal 2007;9:2087-2098.

[25] Johnson J, Maher P, Hanneken A. The flavonoid, eriodictyol, induces long-term protection in ARPE-19 cells through its effects on Nrf2 activation and phase 2 gene expression. Invest Ophthalmol Vis Sci 2009;50:2398-2406.

[26] Chernova T, Nicotera P, Smith AG. Heme deficiency is associated with senescence and causes suppression of N-methyl-D-aspartate receptor subunits expression in primary cortical neurons. Mol Pharmacol 2006;69:697-705.

[27] Jaronczyk K, Bui L, Soong JM, McLaughlin BE, Marks GS, Brien JF, Nakatsu K. The source of heme for vascular heme oxygenase II: de novo heme biosynthesis in rat aorta. Can J Physiol Pharmacol 2004;82:218-224.

[28] Wegiel B and Otterbein LE. Go green: the anti-inflammatory effects of biliverdin reductase. Front Pharmacol 2012;3:47-.

[29] Abuarqoub H, Foresti R, Green CJ, Motterlini R. Heme oxygenase-1 mediates the antiinflammatory actions of 2'-hydroxychalcone in RAW 264.7 murine macrophages. Am J Physiol Cell Physiol 2006;290:C1092-C1099.

[30] Motohashi H and Yamamoto M. Nrf2-Keap1 defines a physiologically important stress response mechanism. Trends Mol Med 2004;10:549-557. 
[31] Aguzzi A, Barres BA, Bennett ML. Microglia: scapegoat, saboteur, or something else? Science 2013;339:156-161.

[32] Martin D, Rojo AI, Salinas M, Diaz R, Gallardo G, Alam J, De Galarreta CM, Cuadrado A. Regulation of heme oxygenase-1 expression through the phosphatidylinositol 3kinase/Akt pathway and the Nrf2 transcription factor in response to the antioxidant phytochemical carnosol. J Biol Chem 2004;279:8919-8929.

[33] Alcaraz MJ, Vicente AM, Araico A, Dominguez JN, Terencio MC, Ferrandiz ML. Role of nuclear factor-kappaB and heme oxygenase-1 in the mechanism of action of an antiinflammatory chalcone derivative in RAW 264.7 cells. Br J Pharmacol 2004;142:11911199.

[34] Foresti R, Hoque M, Monti D, Green CJ, Motterlini R. Differential activation of heme oxygenase- 1 by chalcones and rosolic acid in endothelial cells. J Pharmacol Exp Ther 2005;312:686-693.

[35] Kaidery NA, Banerjee R, Yang L, Smirnova NA, Hushpulian DM, Liby KT, Williams CR, Yamamoto M, Kensler TW, Ratan RR, Sporn MB, Beal MF, Gazaryan IG, Thomas B. Targeting Nrf2-mediated gene transcription by extremely potent synthetic triterpenoids attenuate dopaminergic neurotoxicity in the MPTP mouse model of Parkinson's disease. Antioxid Redox Signal 2013;18:139-157.

[36] Zhang DD. Bardoxolone Brings Nrf2-Based Therapies to Light. Antioxid Redox Signal 2013; In Press.

[37] Maines MD and Kappas A. Enzymatic oxidation of cobalt protoporphyrin IX: observations on the mechanism of heme oxygenase action. Biochemistry 1977;16:419423.

[38] Shan Y, Lambrecht RW, Donohue SE, Bonkovsky HL. Role of Bach1 and Nrf2 in upregulation of the heme oxygenase-1 gene by cobalt protoporphyrin. FASEB J 2006;20:2651-2653. 
[39] Cai C, Teng L, Vu D, He JQ, Guo Y, Li Q, Tang XL, Rokosh G, Bhatnagar A, Bolli R. The heme oxygenase 1 inducer (CoPP) protects human cardiac stem cells against apoptosis through activation of the extracellular signal-regulated kinase (ERK)/NRF2 signaling pathway and cytokine release. J Biol Chem 2012;287:33720-33732.

[40] Zelenka J, Muchova L, Zelenkova M, Vanova K, Vreman HJ, Wong RJ, Vitek L. Intracellular accumulation of bilirubin as a defense mechanism against increased oxidative stress. Biochimie 2012;94:1821-1827.

[41] Bani-Hani MG, Greenstein D, Mann BE, Green CJ, Motterlini R. A carbon monoxidereleasing molecule (CORM-3) attenuates lipopolysaccharide- and interferon-gammainduced inflammation in microglia. Pharmacol Rep 2006;58:132-144.

[42] Orozco LD, Bennett BJ, Farber CR, Ghazalpour A, Pan C, Che N, Wen P, Qi HX, Mutukulu A, Siemers N, Neuhaus I, Yordanova R, Gargalovic P, Pellegrini M, Kirchgessner T, Lusis AJ. Unraveling inflammatory responses using systems genetics and gene-environment interactions in macrophages. Cell 2012;151:658-670.

[43] Zhong LM, Zong Y, Sun L, Guo JZ, Zhang W, He Y, Song R, Wang WM, Xiao CJ, Lu D. Resveratrol inhibits inflammatory responses via the mammalian target of rapamycin signaling pathway in cultured LPS-stimulated microglial cells. PLoS One 2012;7:e32195.

[44] He LF, Chen HJ, Qian LH, Chen GY, Buzby JS. Curcumin protects pre-oligodendrocytes from activated microglia in vitro and in vivo. Brain Res 2010;1339:60-69.

[45] Chen Q, Prior M, Dargusch R, Roberts A, Riek R, Eichmann C, Chiruta C, Akaishi T, Abe K, Maher P, Schubert D. A novel neurotrophic drug for cognitive enhancement and Alzheimer's disease. PLoS One 2011;6:e27865. 


\section{FIGURE LEGENDS}

FIGURE 1. Bilirubin production following exposure of BV2 to HO-1 inducers. A) Bilirubin accumulated over time was measured in the supernatant of microglia cells 6 or $24 \mathrm{~h}$ after exposure to different HO-1 inducers $(20 \mu \mathrm{M}){ }^{*}{ }^{*}<P 0.05$ vs. control $6 \mathrm{~h},{ }^{\dagger}<P 0.05$ vs. control 24 h. B) and C) Bilirubin released in the culture medium following co-incubation of HO-1 inducers $(20 \mu \mathrm{M})$ and the heme oxygenase substrate hemin $(\mathrm{H}, 10 \mu \mathrm{M})$ for 6 or $24 \mathrm{~h}$, respectively. Bilirubin was calculated in picomoles/ml medium and is shown here as fold increase over the control. Data are the mean \pm SEM of 4-6 independent experiments per group. ${ }^{*}<P 0.05$ vs. control (CON), ${ }^{\dagger}<P 0.05$ vs. hemin alone (H).

FIGURE 2. Cellular bilirubin production and heme levels in the presence of a heme biosynthesis inhibitor. A) Bilirubin accumulated in the culture supernatant and B), heme content measured following exposure of BV2 for $6 \mathrm{~h}$ to hemin $(\mathrm{H}, 10 \mu \mathrm{M})$ and supercurcumin $(\mathrm{SC}, 20 \mu \mathrm{M})$ or carnosol $(\mathrm{CA}, 20 \mu \mathrm{M})$ in medium containing $10 \%$ or $1 \% \mathrm{FBS}$, in the presence or absence of succinylacetone (SA, $1 \mathrm{mM}$ ). Bilirubin and heme levels were assessed according to spectrophotometric assays described in Materials and Methods. C) HMOX1 mRNA levels after incubation with different inducers and negatives as measured by RT-PCR. Data are the mean \pm SEM of 3-6 independent experiments per group. ${ }^{*}<P 0.05 v s$. control (CON).

FIGURE 3. Small molecules activators do not increase expression of ALAS 1 or BVR. A) ALAS 1 and B), BVR expression were measured by Western Blot after $6 \mathrm{~h}$ incubation of BV2 cells with $20 \mu \mathrm{M}$ supercurcumin (SC), carnosol (CA), dimethyl fumarate (DMF), probucol (PRO) or resveratrol (R). Results represent the mean of 3 independent experiments and the corresponding densitometric analysis, normalized over the levels of $\beta$-actin, is depicted on the right. C) Heme oxygenase activity was determined in cells exposed to 10 different HO-1 inducers $(20 \mu \mathrm{M})$ in the presence or absence of $\mathrm{N}$-acetylcysteine (NAC, $1 \mathrm{mM}$ ) using a biochemical assay well established in our laboratory. NAC partially reduced the increase in heme oxygenase stimulated by the compounds. Heme oxygenase activity was calculated in picomoles bilirubin/mg protein/60 min and expressed in fold increase over the control values. Data are the mean $\pm \mathrm{SEM}$ 
of 4 independent experiments per group. ${ }^{*}<P 0.05$ vs. control (CON), ${ }^{\dagger}<P 0,05$ vs. HO-1 inducer alone.

FIGURE 4. HO-1 inducers affect nitrite production elicited by INF- $\gamma$ in a concentrationdependent manner. Cells were exposed for $24 \mathrm{~h}$ to INF- $\gamma(15 \mathrm{ng} / \mathrm{ml})$ in the presence of increasing concentrations of the compounds $(5,10$ and $20 \mu \mathrm{M})$ and nitrite levels were measured in the supernatant. All small molecule activators of $\mathrm{Nrf} 2 / \mathrm{HO}-1$ tended to decrease nitrite levels stimulated by INF- $\gamma$, especially at the highest concentration tested $(20 \mu \mathrm{M})$. CoPP and sulforaphane (S) were the most effective even at lower concentrations. The Griess reagent was used to determine nitrite production. Data represent the mean \pm SEM of 5-6 independent experiments per group.

FIGURE 5. Effect of negatives on nitrite production elicited by INF- $\gamma$. Cells were exposed for 24 $\mathrm{h}$ to INF- $\gamma(15 \mathrm{ng} / \mathrm{ml})$ in the presence of increasing concentrations $(5,10$ and $20 \mu \mathrm{M})$ of the compounds that did not up-regulate HO-1 and nitrite levels were measured in the supernatant. Eriodictyol (E) and plumbagin (PLU) were the only ones capable of decreasing nitrite levels, although for PLU this effect is likely due to the extreme cell toxicity caused by the compound. The Griess reagent was used to determine nitrite production. Data represent the mean $\pm \mathrm{SEM}$ of 5-6 independent experiments per group.

FIGURE 6. Small molecule activators affect nitrite production elicited by LPS in a concentration-dependent manner. Cells were exposed for $24 \mathrm{~h}$ to LPS $(0.5 \mu \mathrm{g} / \mathrm{ml})$ in the presence of increasing concentrations $(5,10$ and $20 \mu \mathrm{M})$ of the compounds and nitrite levels were measured in the supernatant. All compounds markedly decreased nitrite levels stimulated by LPS. Carnosol (CA), CoPP, sulforaphane (S) and supercurcumin (SC) were the most effective molecules. The Griess reagent was used to determine nitrite production. Data represent the mean \pm SEM of 5-6 independent experiments per group.

FIGURE 7. Effect of negatives on nitrite production elicited by LPS. Cells were exposed for 24 $\mathrm{h}$ to LPS $(0.5 \mu \mathrm{g} / \mathrm{ml})$ in the presence of increasing concentrations $(5,10$ and $20 \mu \mathrm{M})$ of negatives and nitrite levels were measured in the supernatant. Eriodictyol (E) and plumbagin (PLU) were 
markedly decreased nitrite levels, although this effect was likely due to the extreme cell toxicity caused in the case of PLU. The Griess reagent was used to determine nitrite production. Data represent the mean \pm SEM of 5-6 independent experiments per group.

FIGURE 8. Bilirubin production induced by HO-1 inducers is inversely correlated with nitrite levels. A) Correlation analysis between the amount of bilirubin generated by each positive and nitrite levels following co-incubation of BV2 with INF- $\gamma$ and positive compounds. The results show that higher bilirubin production is associated with lower nitrite. B) The same analysis was performed in cells challenged with LPS, showing a similar, albeit not significant, trend.

FIGURE 9. Changes in the production of inflammatory mediators elicited by small molecules after INF- $\gamma$ challenge are partially controlled by the HO-1 and/or Nrf2 genes. BV2 cells were treated with scrambled control shRNA or shRNA for HO-1 or Nrf2 as described in Materials and Methods. Once transfected colonies were selected, cells were stimulated with INF- $\gamma(15 \mathrm{ng} / \mathrm{ml})$ for $24 \mathrm{~h}$ in the presence or absence of the HO-1 inducers tert-butyl hydroquinone (tBH), carnosol (CA), CoPP and dimethyl fumarate (DMF) and the negatives probucol (PRO) and resveratrol (R) at $10 \mu \mathrm{M}$ concentration and the supernatant was collected for measurement of nitrite (Griess reagent) and $\mathrm{PGE}_{2}$ and TNF- $\alpha$ by ELISA. A) Compounds decreased nitrite levels to different extents and silencing HO-1 or Nrf2 partially reversed this effect. B) Knock down of HO-1 or Nrf2 did not reverse the inhibition of $\mathrm{PGE}_{2}$ production by compounds. C) INF- $\gamma$-mediated TNF- $\alpha$ production was partially decreased by compounds but was not reversed in cells silenced for HO-1 or Nrf2. Data are the mean \pm SEM of 3 independent experiments per group. $*<P 0.05$ vs. unstimulated cells (CON), ${ }^{\dagger}<P 0.05$ vs. scrambled-transfected cells, ${ }^{\#} P 0.05$ vs. cells stimulated with INF- $\gamma$ only.

FIGURE 10. Changes in the production of inflammatory mediators elicited by small molecules after LPS challenge are partially controlled by the HO-1 and/or Nrf2 genes. BV2 cells were treated with scrambled control shRNA or shRNA for HO-1 or Nrf2 as described in Materials and Methods. The same treatments and measurements described for Fig. 9 were applied in cells stimulated with LPS $(0.5 \mu \mathrm{g} / \mathrm{ml})$ for $24 \mathrm{~h}$. A) Compounds decreased nitrite levels to different extents and silencing Nrf2 partially reversed this effect. B) Knock down of HO-1 or Nrf2 reduced 
$\mathrm{PGE}_{2}$ levels induced by LPS and did not reverse the inhibition of $\mathrm{PGE}_{2}$ production by compounds. C) LPS-mediated TNF- $\alpha$ production was increased in cells silenced for HO-1 or Nrf2. Compounds partially decreased TNF- $\alpha$ levels and silencing HO-1 partially reversed this effect. Data are the mean \pm SEM of 3 independent experiments per group. ${ }^{*}<P 0,05$ vs unstimulated cells (CON), ${ }^{\dagger}<P 0.05$ vs. scrambled-transfected cells, ${ }^{\#} P 0.05$ vs. cells stimulated with LPS only.

Supplementary Figure 1. Inhibition of heme oxygenase activity by SnPPIX partially reverses the decrease in INF- $\gamma$-mediated nitrite production exerted by some HO-1 inducers. SnPPIX (10 $\mu \mathrm{M})$, the inhibitor of heme oxygenase activity, was co-incubated with small molecule activators $(10 \mu \mathrm{M})$ in cells challenged with INF- $\gamma(15 \mathrm{ng} / \mathrm{ml})$ for $24 \mathrm{~h}$. We observed that the decrease in nitrite levels elicited by tert-butyl hydroquinone (tBH), carnosol (CA), CoPP, supercurcumin (SC) and sulforaphane (S) was partially reversed by SnPPIX. Nitrite was measured in the cell supernatant using the Griess reagent. Data represent the mean \pm SEM of 5-6 independent experiments per group.

Supplementary Figure 2. Small contribution of the HO-1 pathway in the decrease of nitrite production exerted by certain compounds in LPS-challenged BV2 cells. SnPPIX $(10 \mu \mathrm{M})$ was coincubated with small molecule activators $(10 \mu \mathrm{M})$ in cells challenged with LPS $(0.5 \mu \mathrm{g} / \mathrm{ml})$ for $24 \mathrm{~h}$. The heme oxygenase inhibitor modestly reversed the decrease in nitrite levels elicited by tert-butyl hydroquinone (tBH), carnosic acid (CAA), carnosol (CA), and isoliquiritigenin (ISL). Nitrite was measured in the cell supernatant using the Griess reagent. Data represent the mean \pm SEM of 5-6 independent experiments per group. 
Figure 1

Click here to download high resolution image

A



B
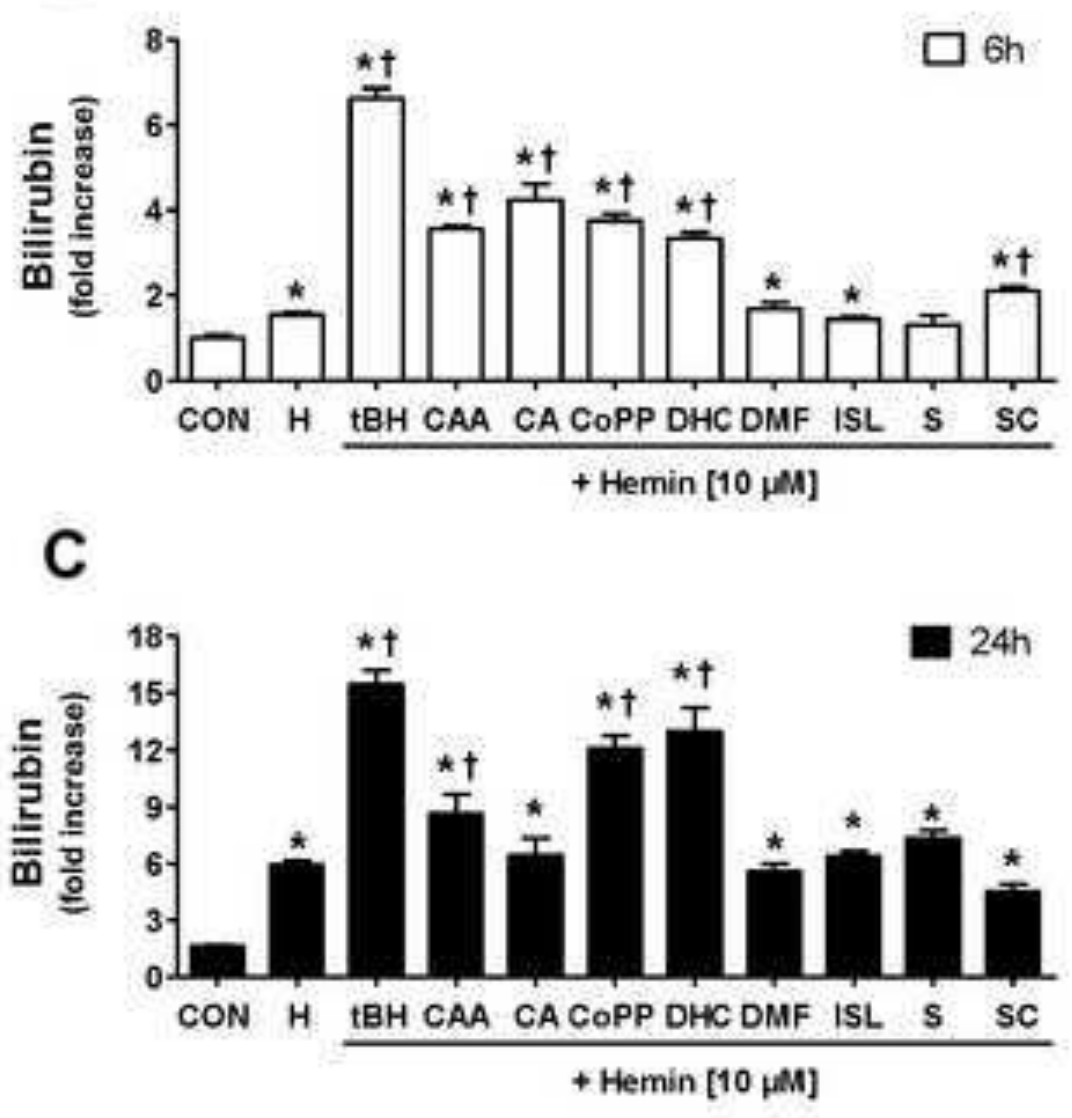
Figure 2

A

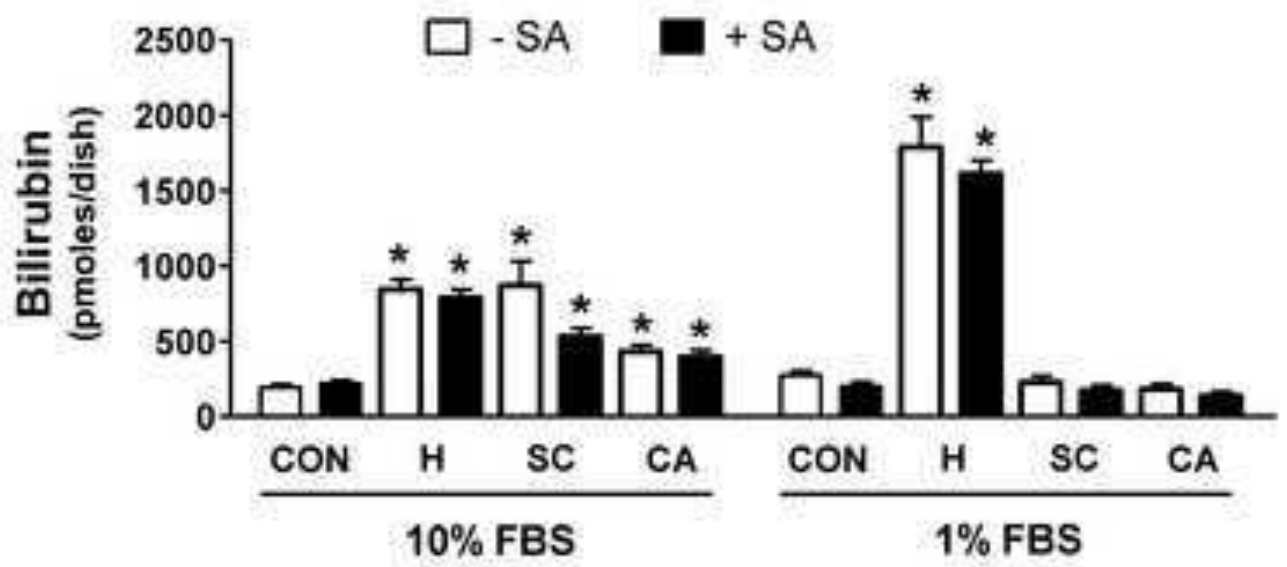

B

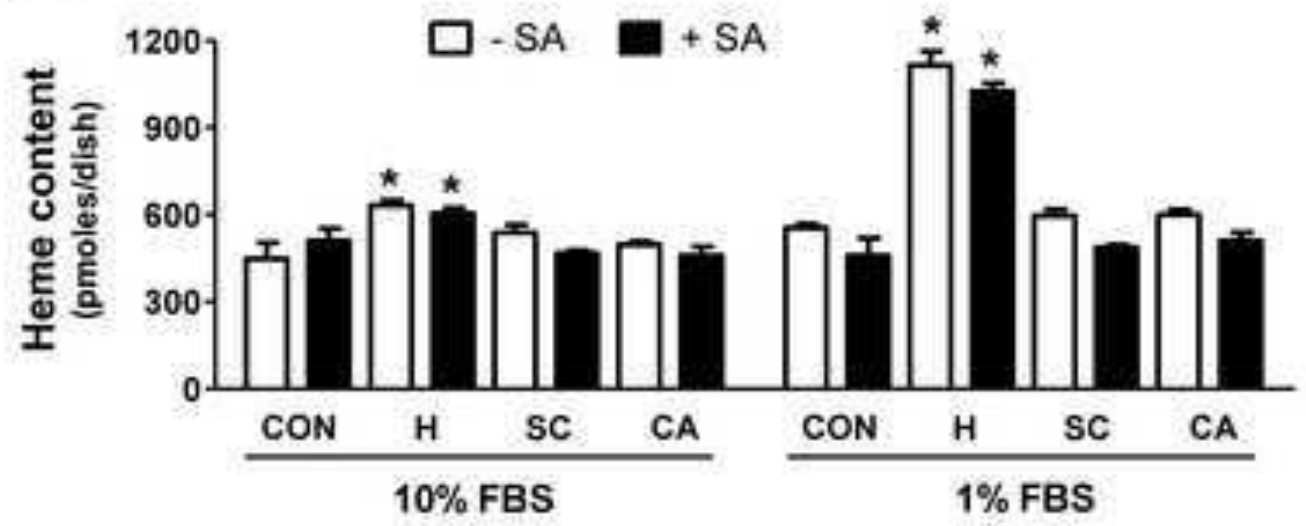

C

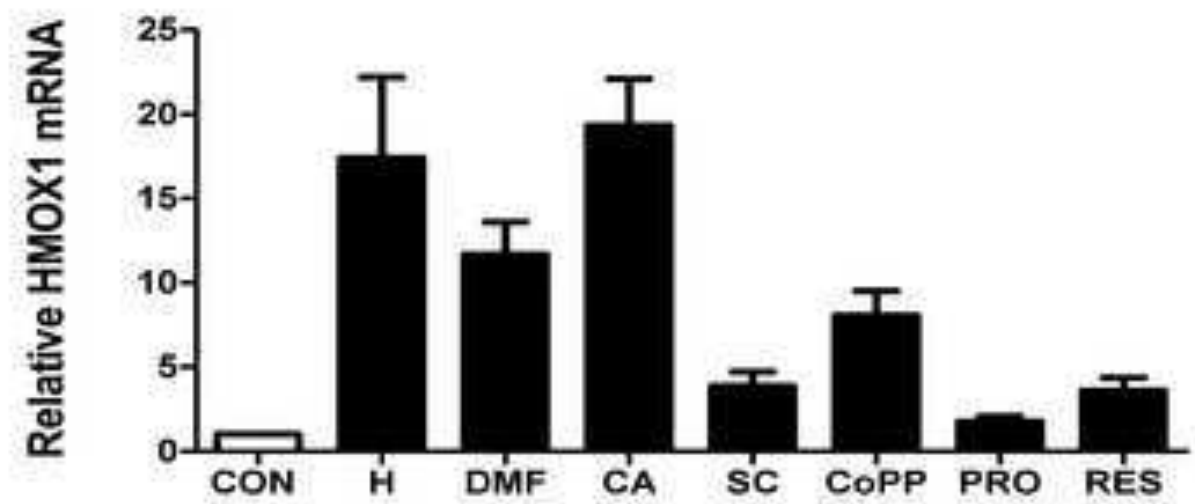


Figure 3

\section{A}

$65 \mathrm{kDa}$

ALAS 1

$\beta$-actin

CON SC CA DMF PR R

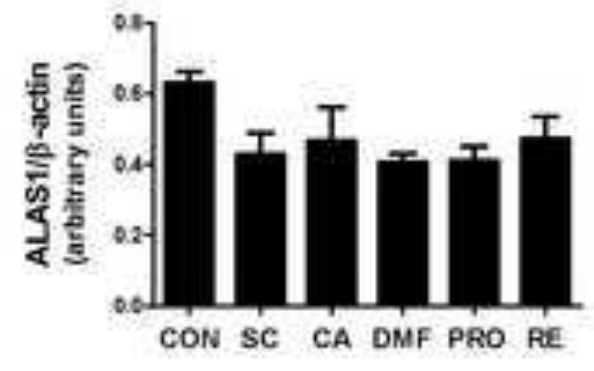

B

$40 \mathrm{kDa}$

BVR

$\beta$-actin

CON SC CA DMF PR R

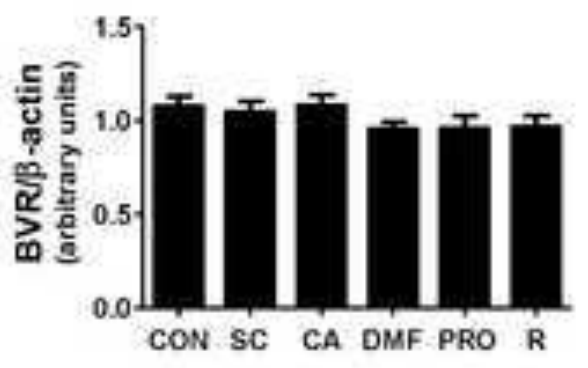

C

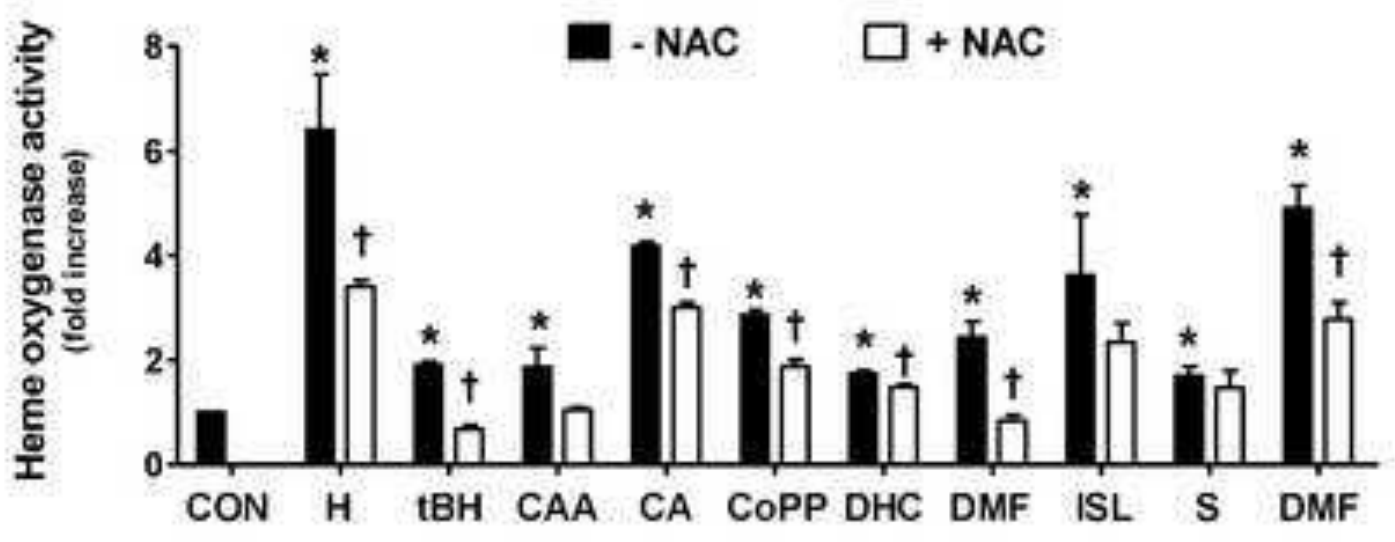



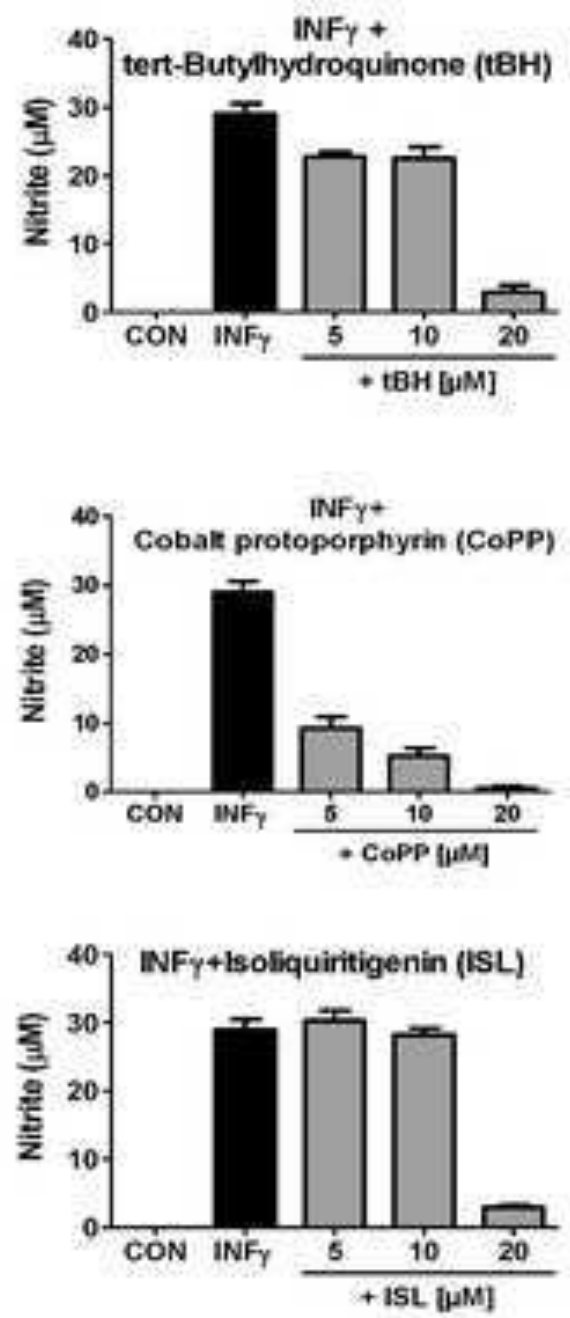
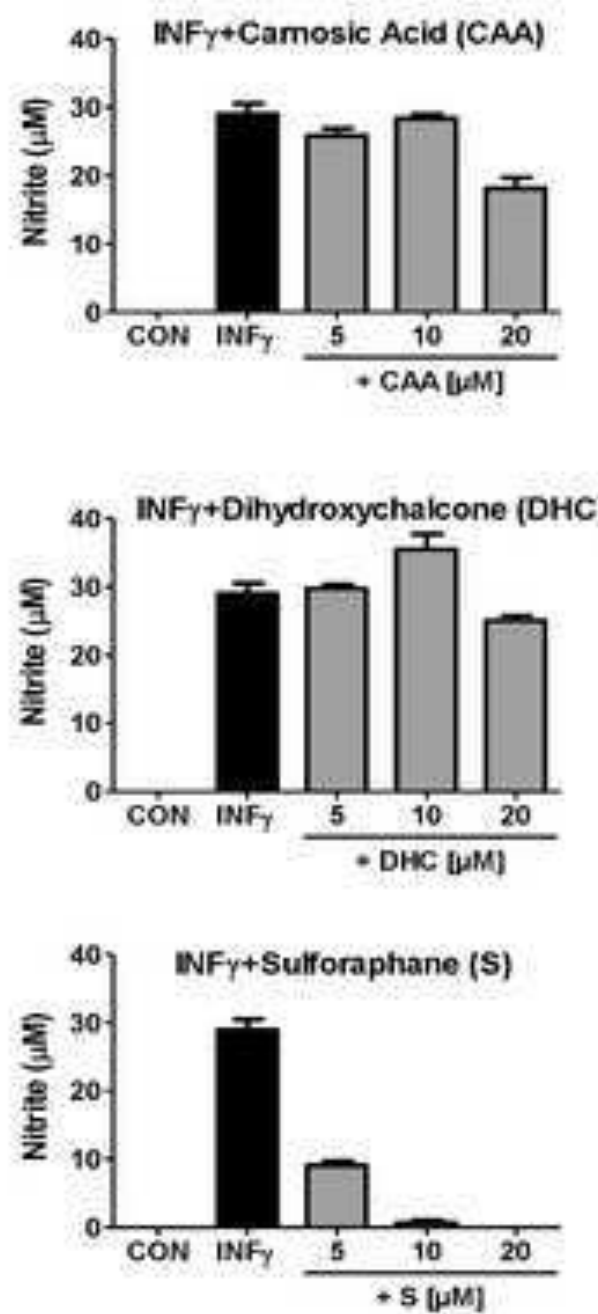
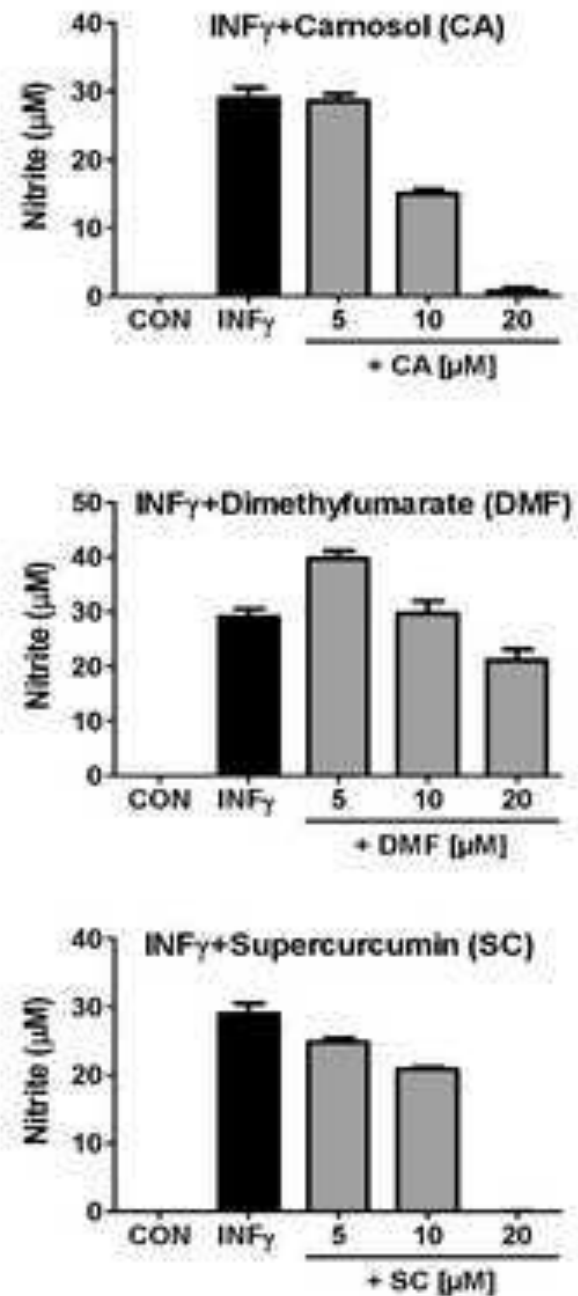


\section{Figure 5}

Click here to download high resolution image
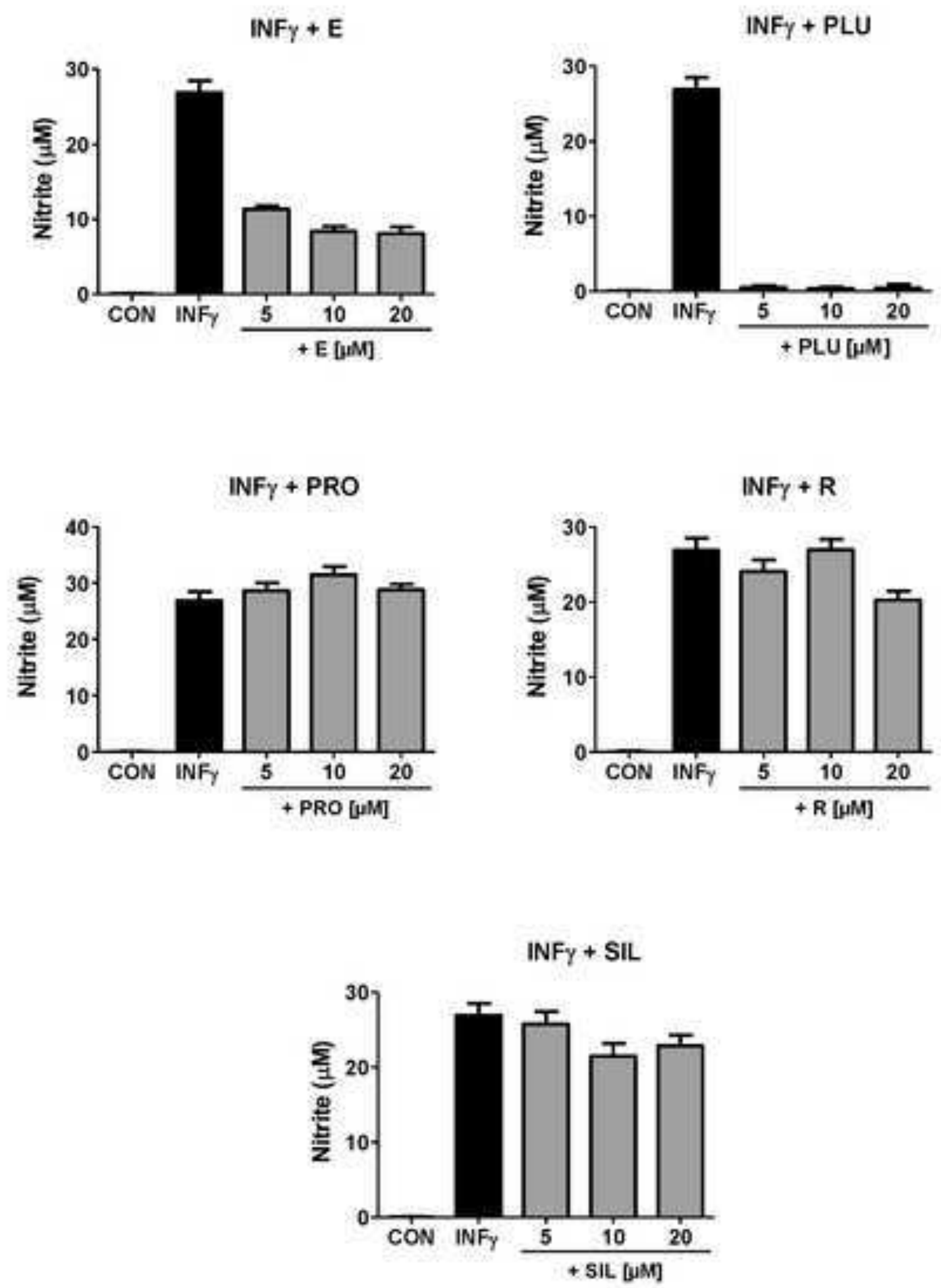
LPS+

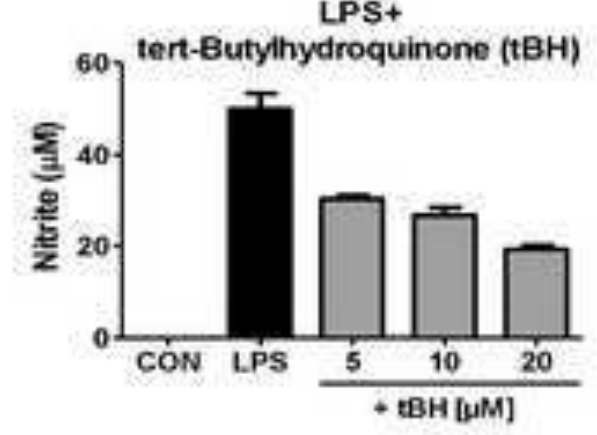

LPS+
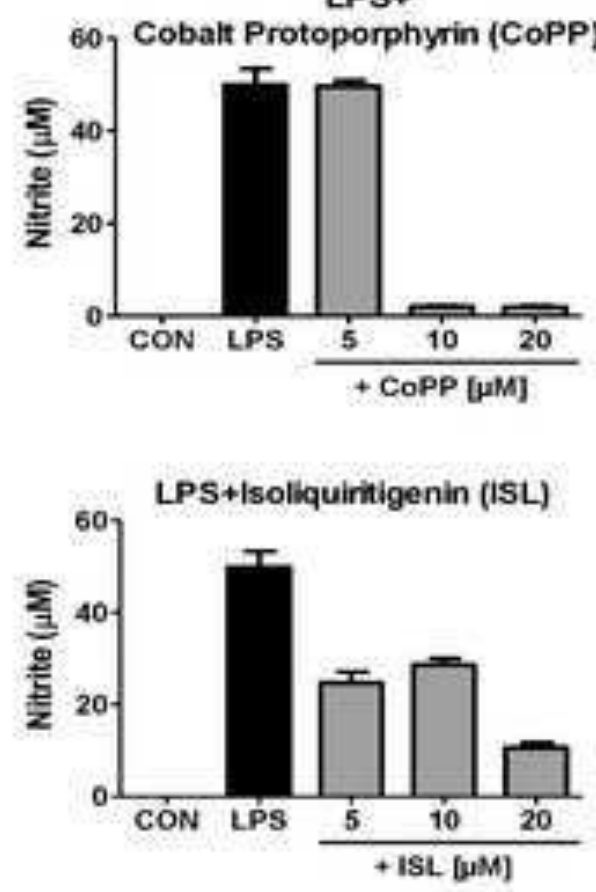




\section{Figure 7}

Click here to download high resolution image
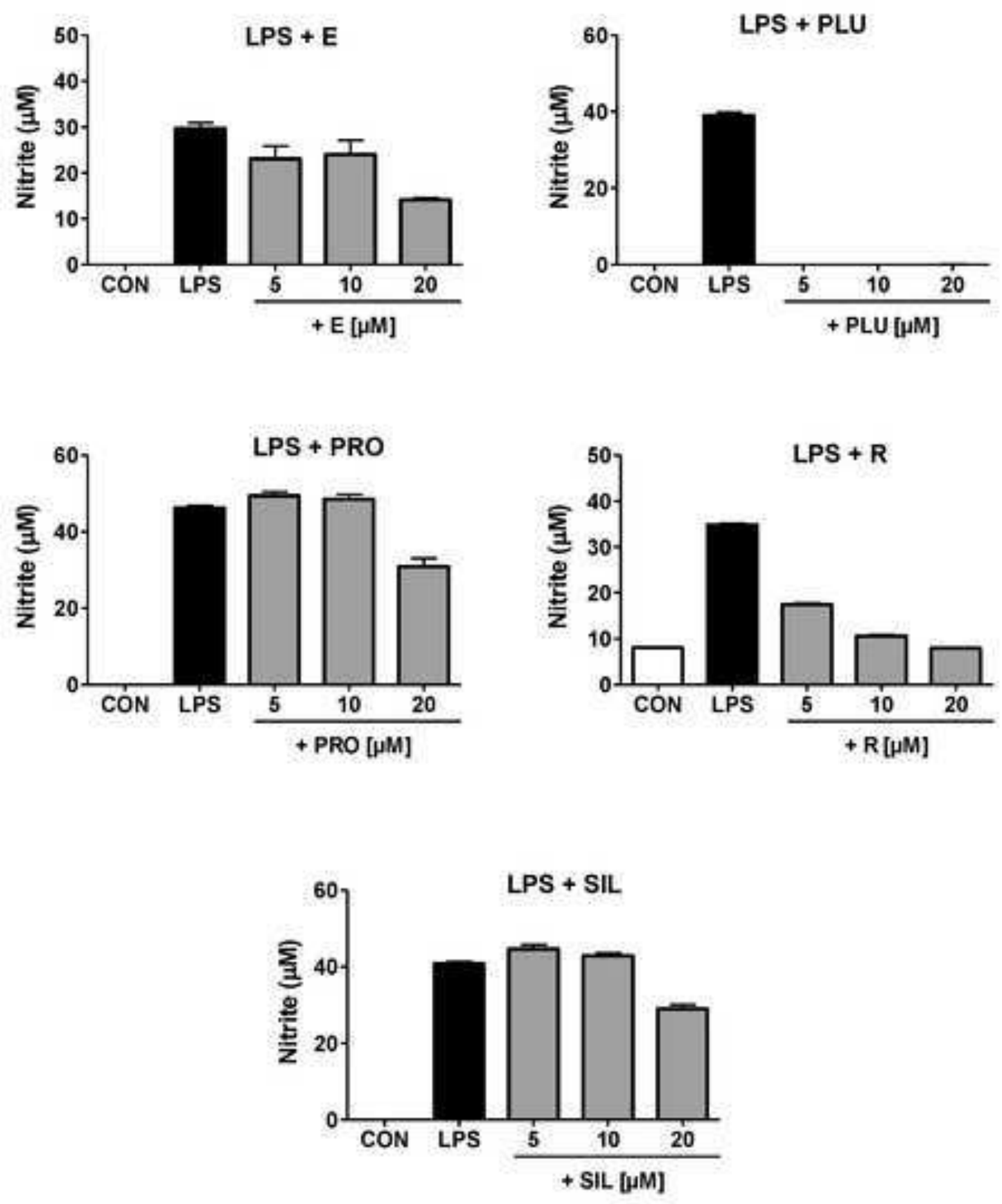
Figure 8
Click here to download high resolution image

A



B

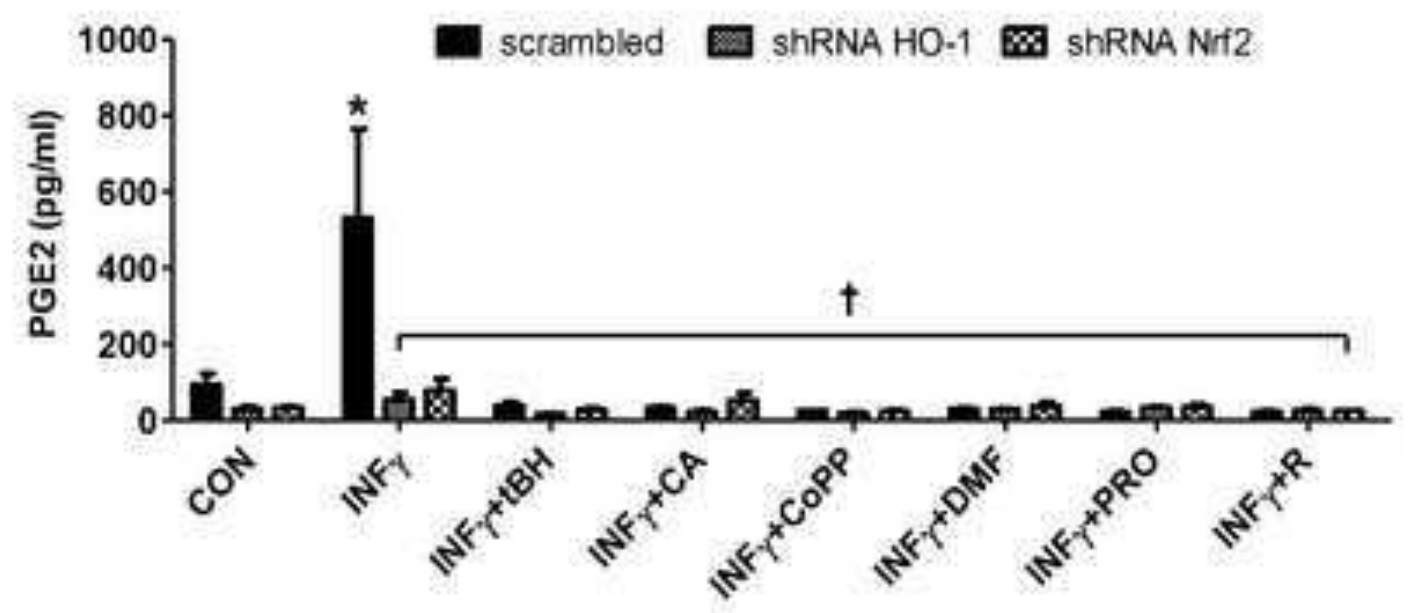

C

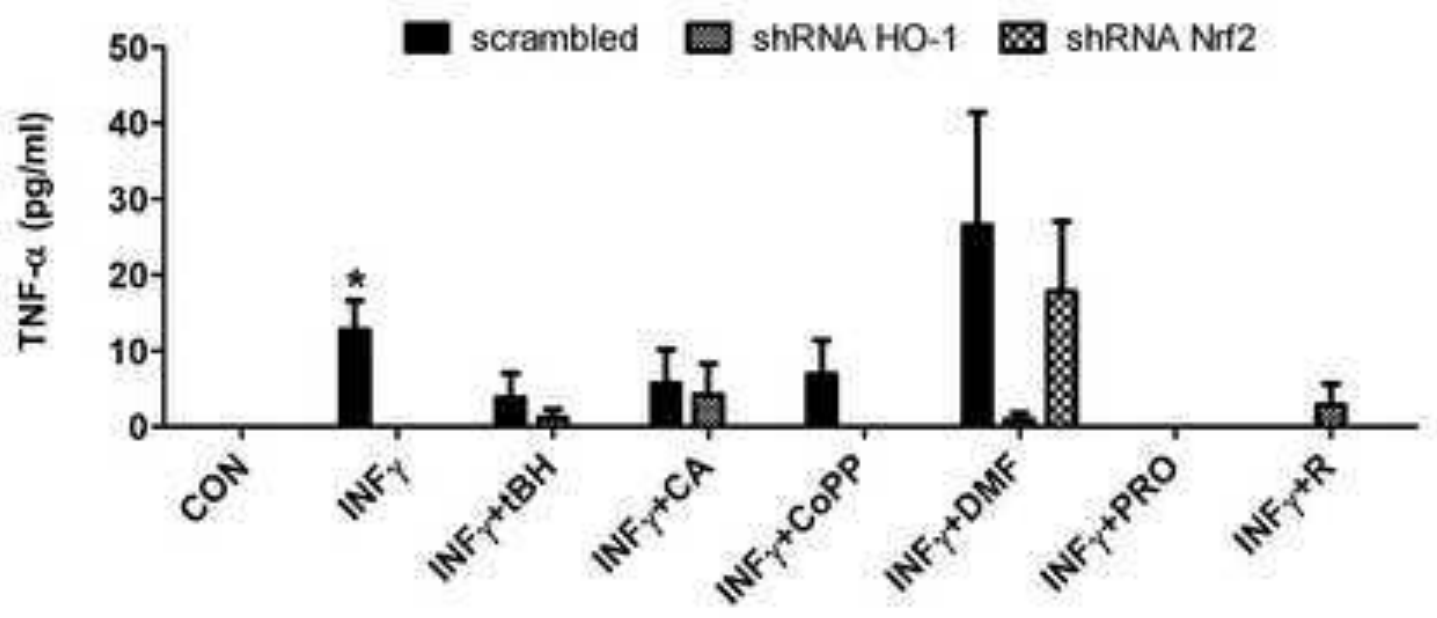


A

LPS scrambled $\square$ shRNAHO-1 $\mathrm{W}$ shRNA Nrt2

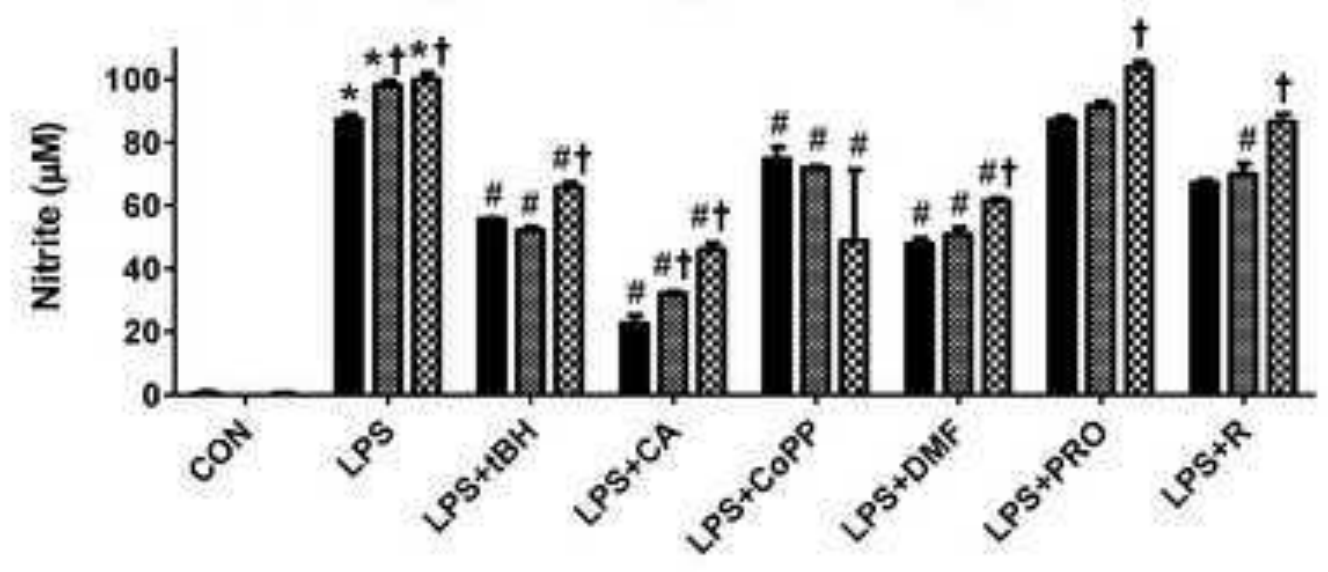

B
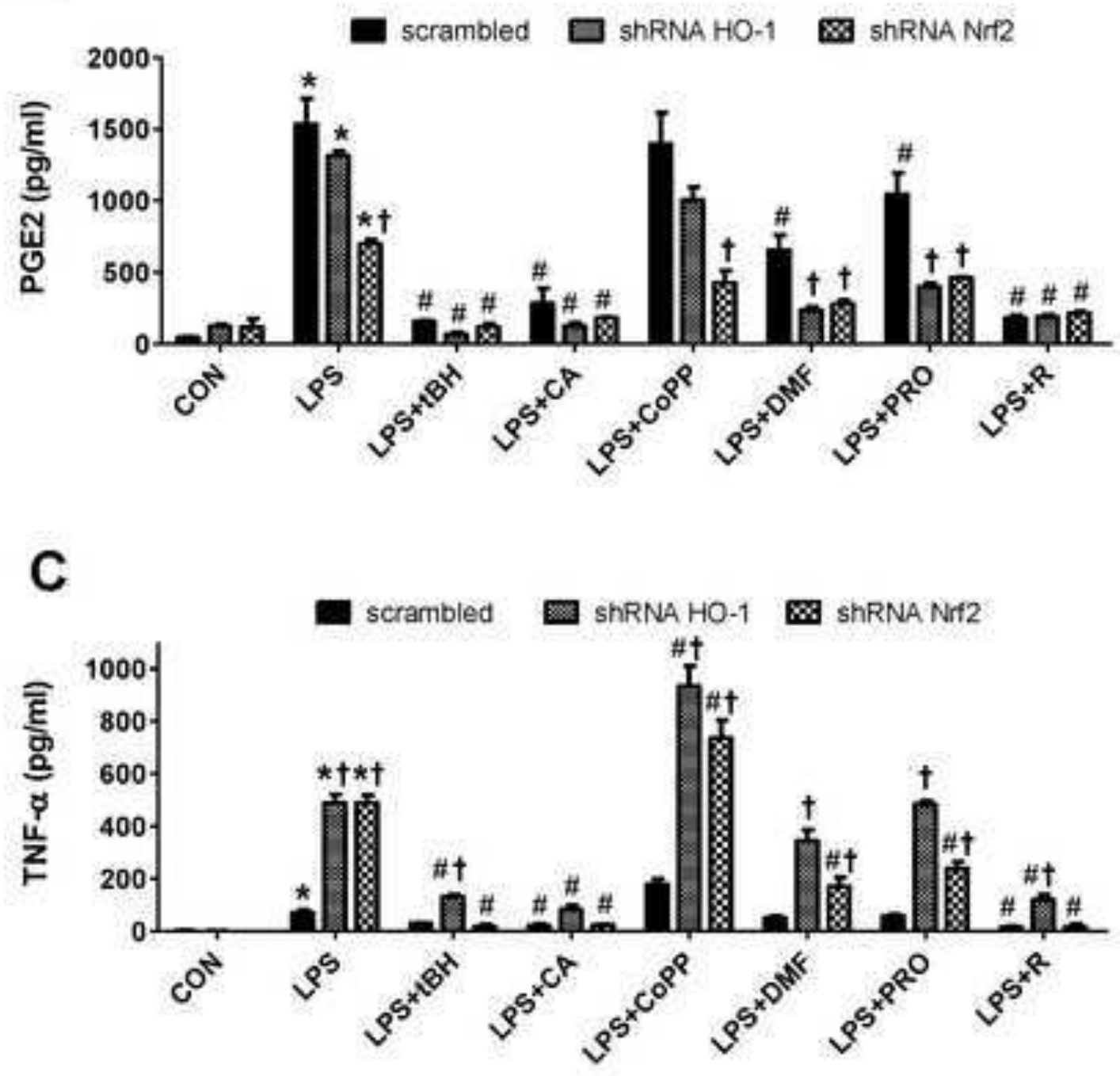
Table 1. List of compounds tested in the initial screening.

\begin{tabular}{|c|c|c|c|c|}
\hline COMPOUND & $\begin{array}{c}\text { HO }-1 \text { induction } \\
6 \mathrm{~h}\end{array}$ & $\begin{array}{c}\text { HO -1 induction } \\
24 \mathrm{~h}\end{array}$ & $\begin{array}{c}\text { Cytotoxicity } \\
(\geq 15 \% \text { LDH } \\
\text { release })^{\mathrm{a}}\end{array}$ & Solubility \\
\hline Acetaminophen $^{\mathrm{b}}$ & - & - & - & $\mathrm{DMSO}^{\mathrm{c}}$ \\
\hline Acetylcarnitine & - & - & - & $\mathrm{H}_{2} \mathrm{O}$ \\
\hline Allyl isothiocyanate & $\uparrow^{d}$ & $\uparrow$ & $\uparrow d$ & DMSO \\
\hline Allyl sulfide & - & - & - & $\mathrm{H}_{2} \mathrm{O}$ \\
\hline Auronafin & $\uparrow$ & $\uparrow$ & $\uparrow$ & DMSO \\
\hline Aurothioglucose hydrate & - & - & - & $\mathrm{H}_{2} \mathrm{O}$ \\
\hline $\begin{array}{l}\text { Aurothiomalate } \\
\text { (sodium salt) }\end{array}$ & - & - & - & DMSO \\
\hline Baicalein & - & - & - & DMSO \\
\hline tert-Butylhydroquinone & $\uparrow$ & $\uparrow$ & $\uparrow$ & DMSO \\
\hline $\begin{array}{l}\text { Caffeic acid } \\
\text { phenethyl ester }\end{array}$ & $\uparrow$ & $\uparrow$ & $\uparrow$ & DMSO \\
\hline Capsaicin & - & - & - & DMSO \\
\hline Carnosic acid & $\uparrow$ & $\uparrow$ (only at $5 \mu \mathrm{M}$ ) & - & DMSO \\
\hline Carnosol & $\uparrow$ & $\uparrow$ & - & DMSO \\
\hline Celastrol & $\uparrow($ only at $5 \mu \mathrm{M})$ & $\uparrow$ (only at $5 \mu \mathrm{M}$ ) & - & DMSO \\
\hline Celecoxib & $\uparrow$ & - & - & DMSO \\
\hline $\begin{array}{l}\text { Chlorophyllin } \\
\text { (sodium copper salt) }\end{array}$ & - & - & - & $\mathrm{H}_{2} \mathrm{O}$ \\
\hline Cilostazol & - & - & - & DMSO \\
\hline trans-Cinnamaldehyde & - & - & - & $\mathrm{H}_{2} \mathrm{O}$ \\
\hline Cobalt protoporphyrin IX & $\uparrow$ & $\uparrow$ & - & $0.01 \mathrm{M} \mathrm{NaOH}$ \\
\hline Costunolide & - & - & - & DMSO \\
\hline Dehydrocostus lactone & $\uparrow$ & $\uparrow$ & $\uparrow$ & DMSO \\
\hline Deltamethrin & - & - & - & DMSO \\
\hline 2,2-Dihydroxychalcone & $\uparrow$ & $\uparrow$ & $\uparrow$ & DMSO \\
\hline Dimethyl fumarate & $\uparrow$ & $\uparrow$ & - & $\mathrm{H}_{2} \mathrm{O}$ \\
\hline Enterolactone & - & - & - & DMSO \\
\hline Epibatidine & - & - & $\uparrow$ & DMSO \\
\hline Epigallocatechin & $\uparrow($ only at $5 \mu \mathrm{M})$ & - & - & $\mathrm{H}_{2} \mathrm{O}$ \\
\hline Epigallocatechin gallate & - & - & - & $\mathrm{H}_{2} \mathrm{O}$ \\
\hline Eriodictyol & - & - & - & DMSO \\
\hline Ferulic acid & - & - & - & DMSO \\
\hline Flunarazine & - & - & - & DMSO \\
\hline $\begin{array}{l}\text { Hemin } \\
\text { (internal positive) }\end{array}$ & $\uparrow$ & not tested & not tested & $0.01 \mathrm{M} \mathrm{NaOH}$ \\
\hline Hesperidin & - & - & - & DMSO \\
\hline 2-Hydroxychalcone & $\uparrow$ (only at $20 \mu \mathrm{M}$ ) & - & - & DMSO \\
\hline Isoliquiritigenin & $\uparrow$ & - & $\uparrow$ & DMSO \\
\hline Kaempferol & - & - & - & DMSO \\
\hline Lansoprazole & - & - & $\uparrow$ & DMSO \\
\hline$\alpha$-Lipoic acid & $\uparrow($ only at $5 \mu \mathrm{M})$ & $\uparrow$ (only at $5 \mu \mathrm{M}$ ) & - & DMSO \\
\hline Melatonin & - & - & - & DMSO \\
\hline Nicotine & $\uparrow($ only at $5 \mu \mathrm{M})$ & - & - & DMSO \\
\hline
\end{tabular}




\begin{tabular}{lllll} 
Nordihydroguaiaretic & $\uparrow$ & $\uparrow$ & $\uparrow$ & DMSO \\
acid & - & - & - & DMSO \\
Piceatannol & - & - & $\uparrow$ & DMSO \\
Piperine & - & - & - & DMSO \\
Plumbagin & - & - & $\uparrow$ & DMSO \\
Probucol & - & - & $\uparrow$ & DMSO \\
Puerarin & - & - & - & DMSO \\
Quercitin & - & - & - & DMSO \\
Resveratrol & - & - & - & DMSO \\
Rottlerin & - & - & - & DMSO \\
Sildenafil & - & - & - & DMSO \\
trans-Stilbene oxide & - & - & DMSO \\
Sulfasalazine & - & $\uparrow$ & - & DMSO \\
Sulforaphane & $\uparrow$ & - & - & DMSO \\
Supercurcumin & - & - & DMSO \\
Tangeretin & - & - & $\uparrow$ & DMSO \\
Tanshinone & $\uparrow$ & & DMSO \\
2,2,4-Trihydroxychalcone & & - & & \\
\hline
\end{tabular}

\footnotetext{
${ }^{\mathrm{a}} \mathrm{LDH}$, lactate dehydrogenase release assay

b Fifty-six compounds were selected from over 100 molecules identified through a literature search as HO-1 and/or Nrf2 inducers

${ }^{\mathrm{c}}$ DMSO, dimethyl sulfoxide

${ }^{\mathrm{d}}$ Arrows indicate an increased HO-1 protein expression at the time indicated or a cytotoxicity $\geq$ $15 \%$ when cells were exposed for $24 \mathrm{~h}$ to the compounds listed at $20 \mu \mathrm{M}$ final concentration
} 
Table 2. Chemical structure and potency of HO-1 inducers (positive compounds).

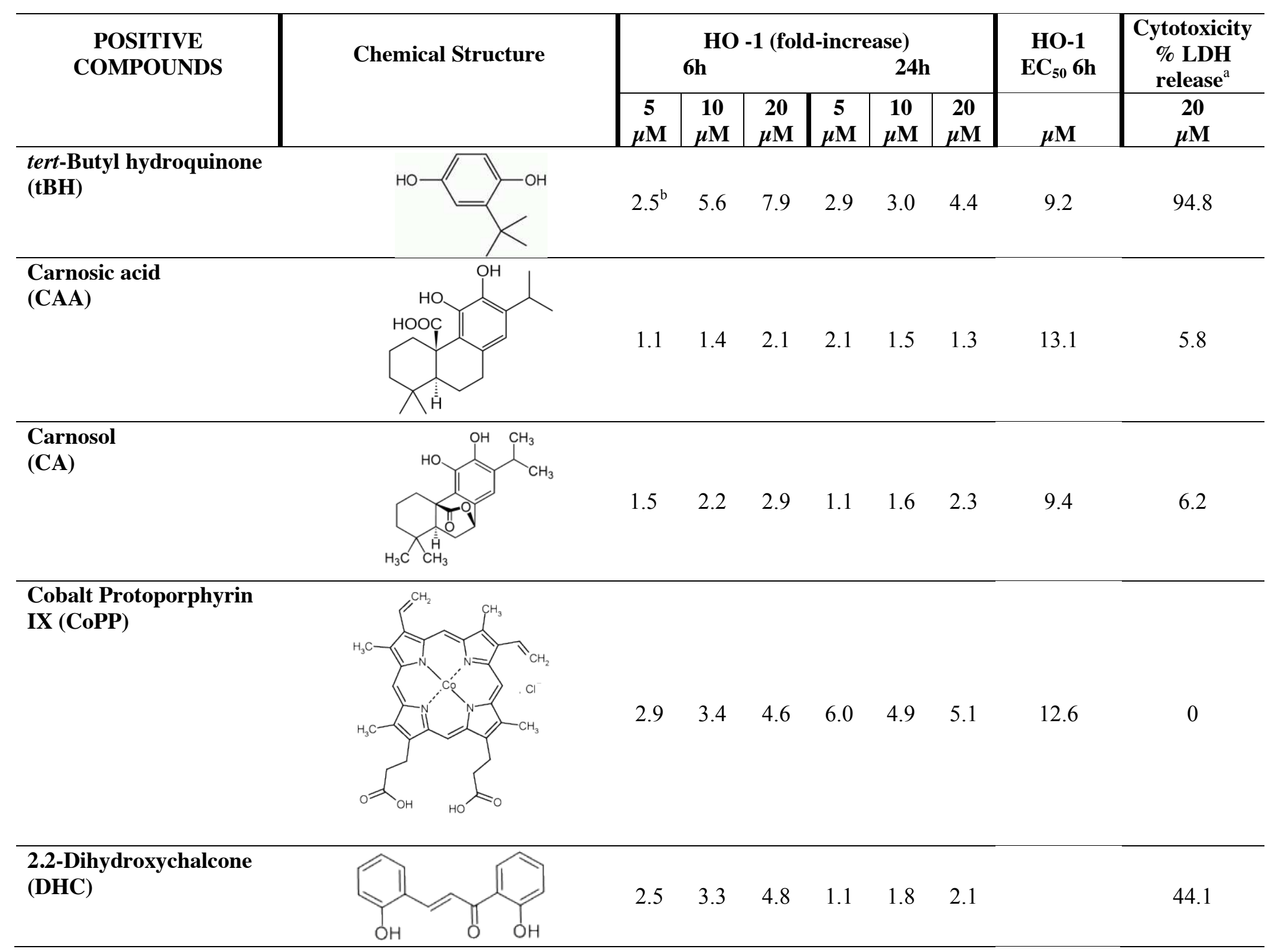




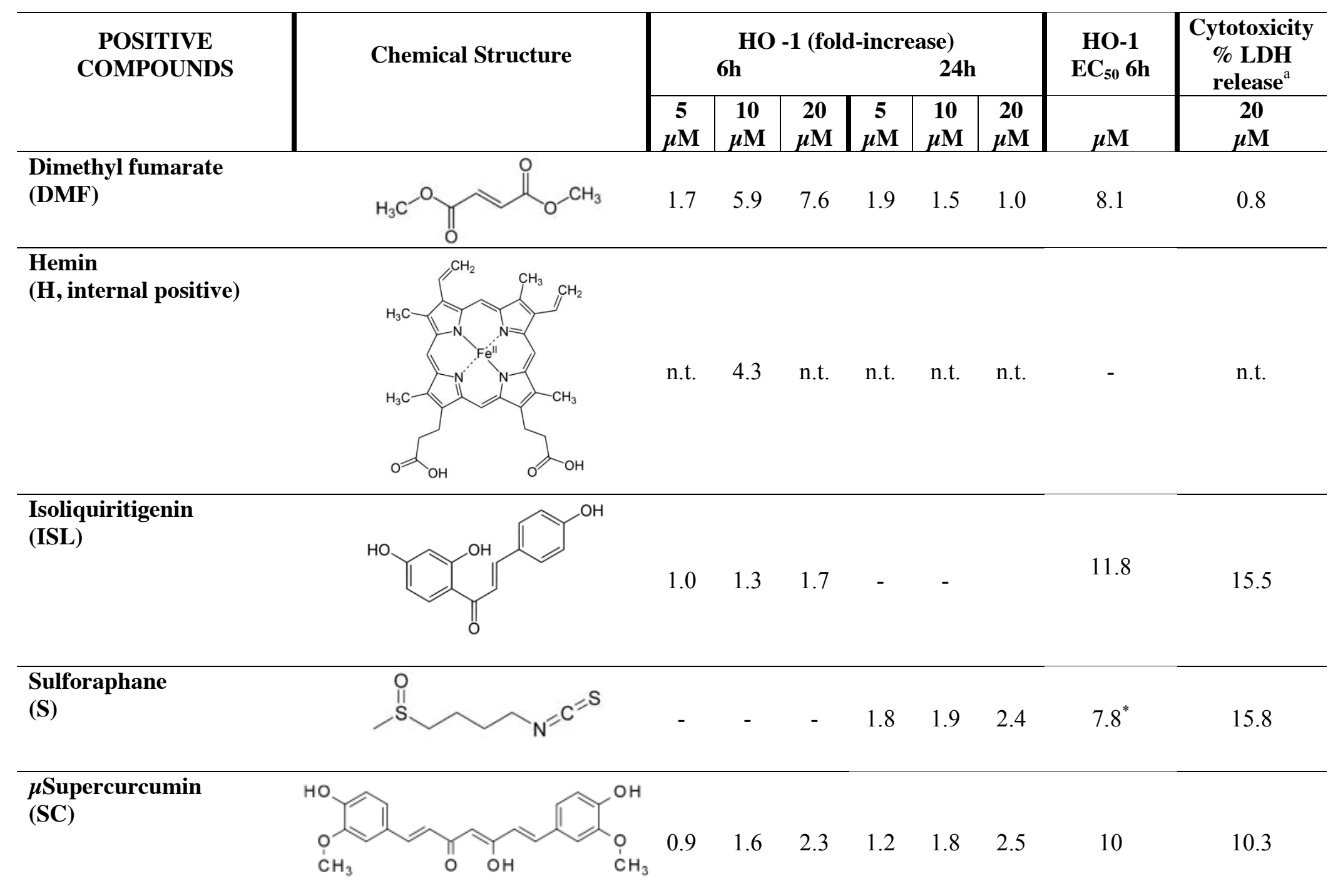

\footnotetext{
${ }^{a}$ Cytotoxicity, expressed as \% of lactate dehydrogenase (LDH) release, was measured after incubation of BV2 microglia cells for $24 \mathrm{~h}$ with $20 \mu \mathrm{M}$ of the indicated compounds.
} 
${ }^{\mathrm{b}}$ HO-1 protein expression was measured as fold-increase at 6 and $24 \mathrm{~h}$ after treatment of BV2 microglia cells with compounds at the concentrations indicated. 
Table 3. Chemical structure and cytotoxicity of compounds not inducing HO-1 (negative compounds).

$\begin{aligned} & \text { NEGATIVE } \\ & \text { COMPOUNDS }\end{aligned}$
Resveratrol
R)

${ }^{a}$ Cytotoxicity, expressed as \% of lactate dehydrogenase (LDH) release, was measured after incubation of BV2 microglia cells for $24 \mathrm{~h}$ with $20 \mu \mathrm{M}$ of the indicated compounds. 
INF $\gamma(15 \mathrm{ng} / \mathrm{ml})+$ Positives $(10 \mu M)+\operatorname{SnPPIX}(10 \mu M)$
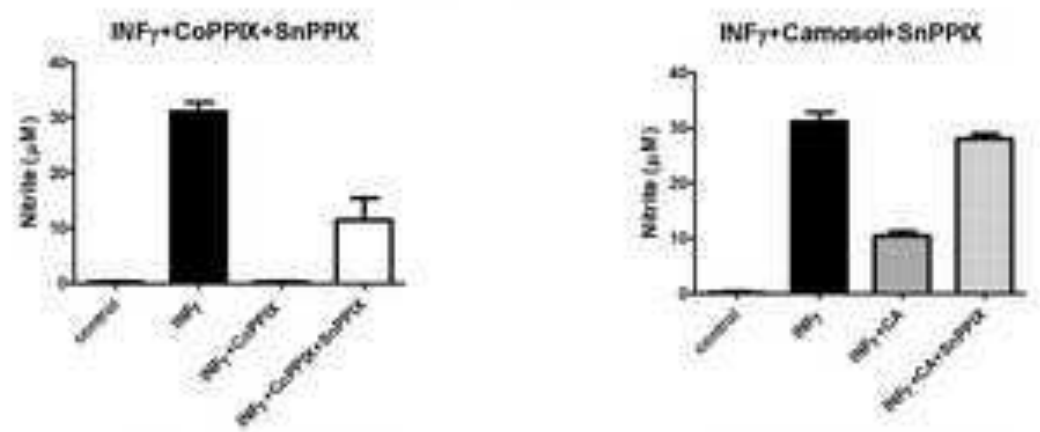

INF- Supercureumin+\$nPPQ

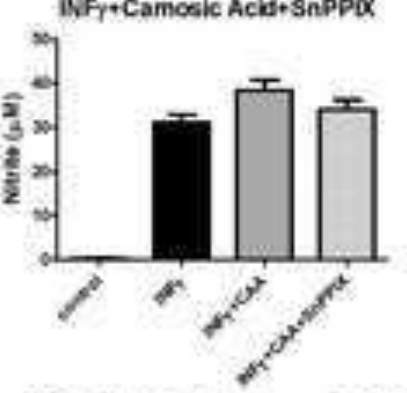

WFF+Sultoraphane+SnPPIX

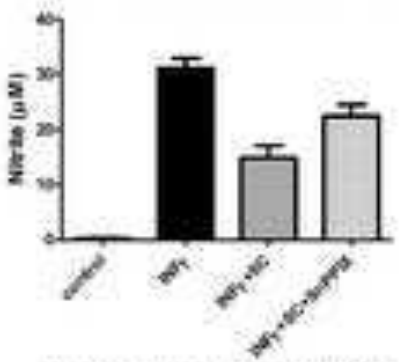

WFy Dhydrochakone+sinPpox
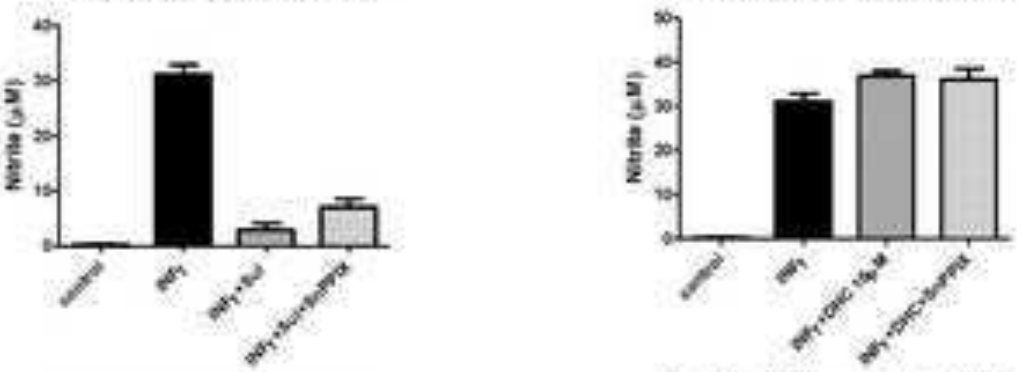

WF + Tbutyeydroquinone+SnPPD

INFy+botquirtigenin+SnPPX

WF, Dimethythumarate+SnPPO
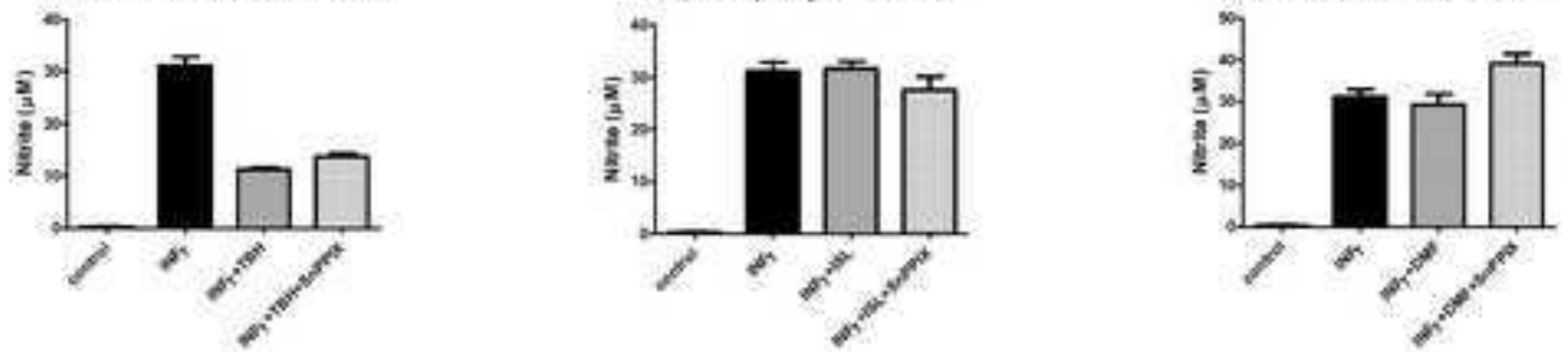
LPS $(0,5 \mu \mathrm{g} / \mathrm{ml})+$ POSITIVES $(10 \mu \mathrm{M})+\operatorname{SnPPIX}(10 \mu \mathrm{M})$
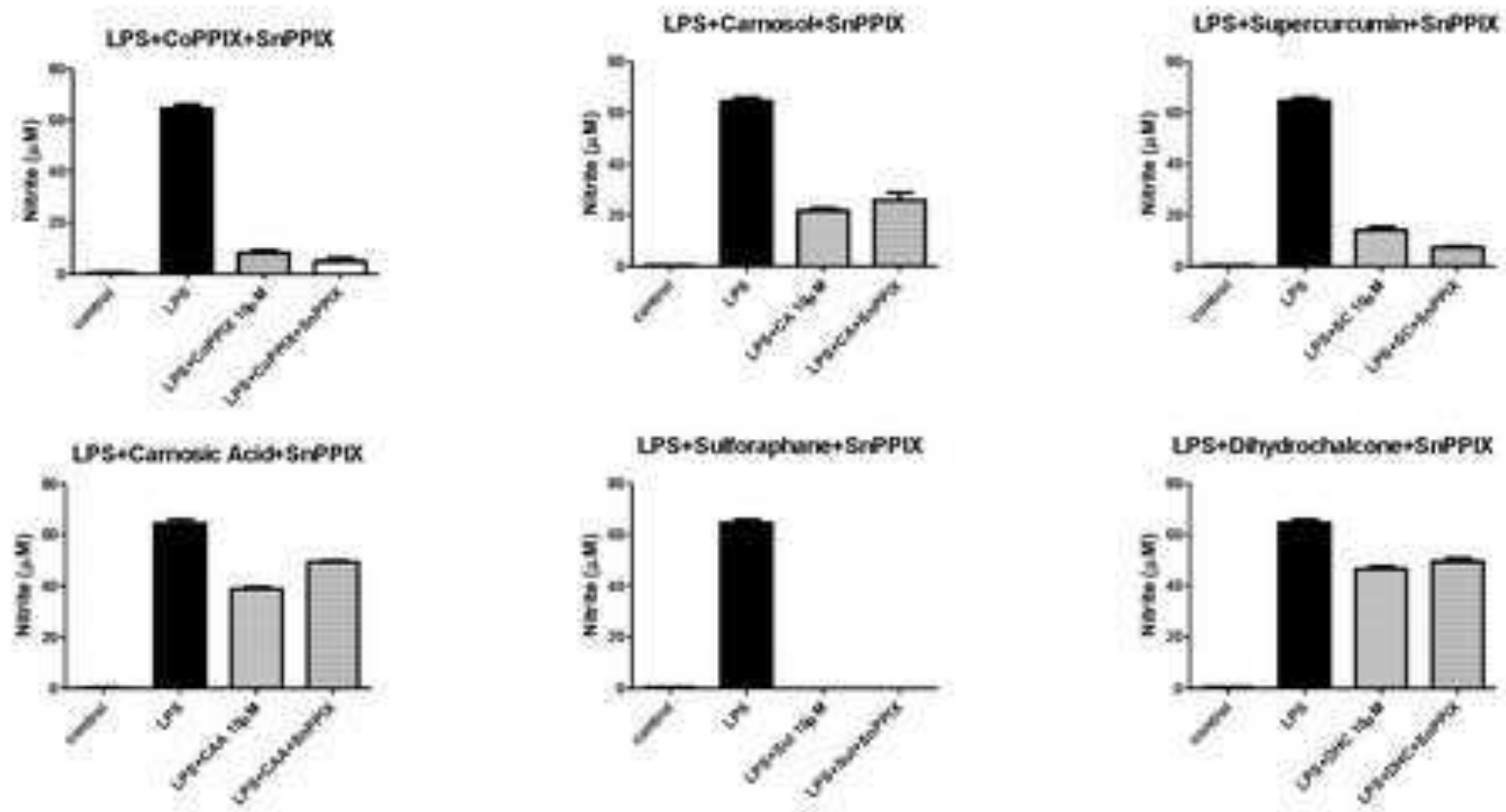

UPS+OErydrochakone+SIPPIX

LP- Tbutyendroquanonesinp $\alpha x$
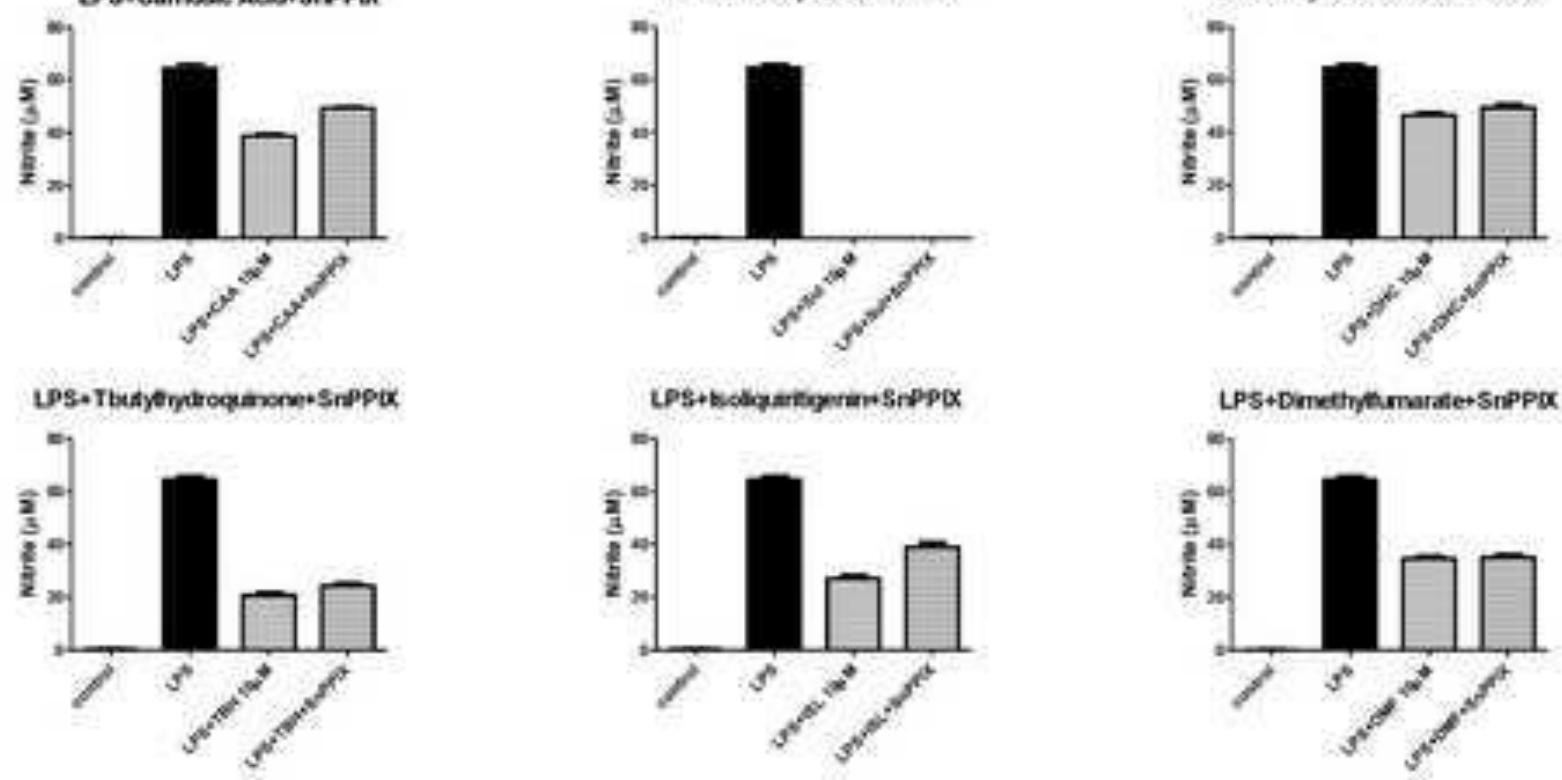


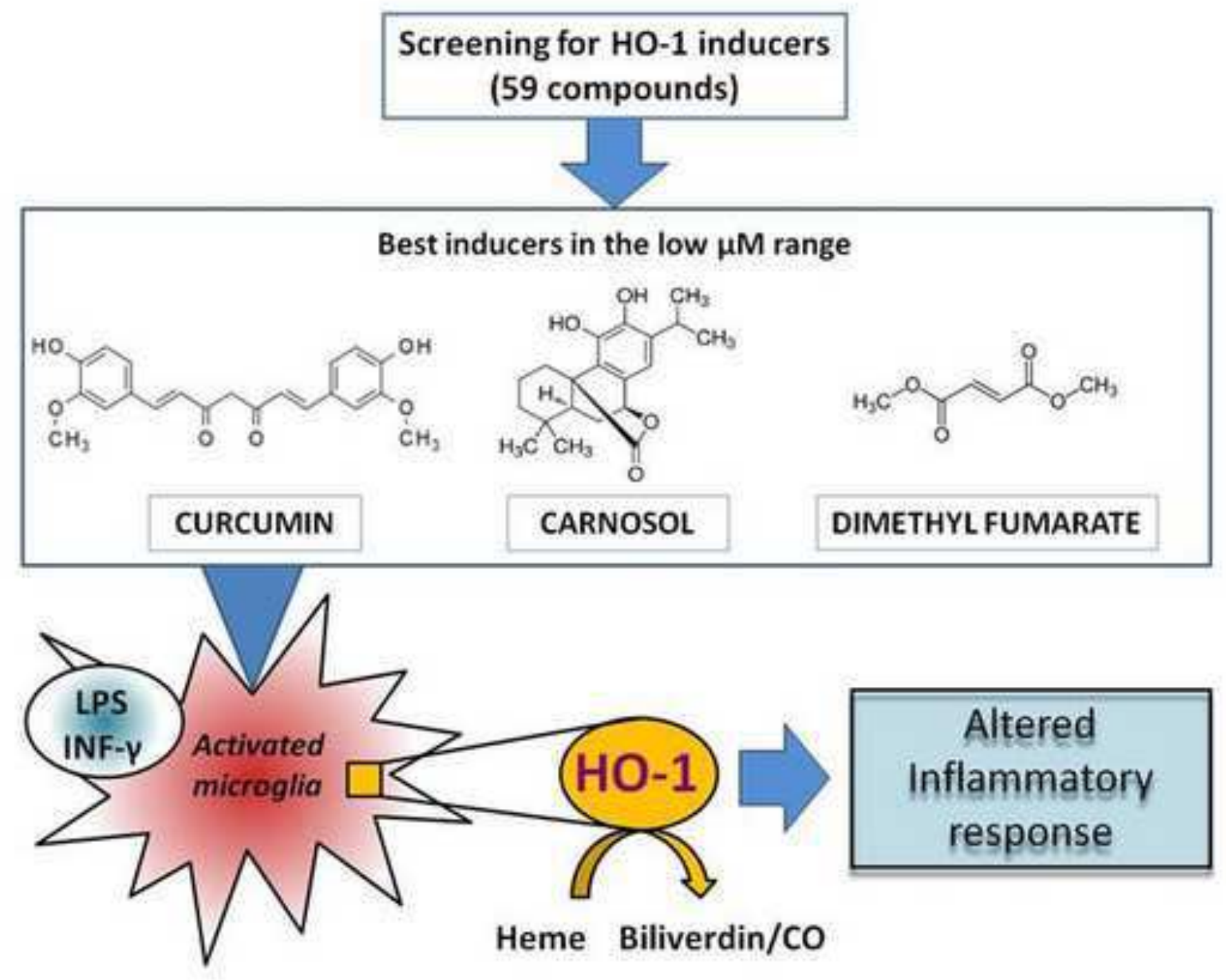

